\title{
ARTICLE Alcohol-induced IL-17A production in Paneth cells amplifies endoplasmic reticulum stress, apoptosis, and inflammasome- IL-18 activation in the proximal small intestine in mice
}

\author{
B. Gyongyosi ${ }^{1}$, Y. Cho ${ }^{1}$, P. Lowe ${ }^{1}$, C. D. Calenda ${ }^{1}$, A. Iracheta-Vellve ${ }^{1}$, A. Satishchandran ${ }^{1}$, A. Ambade ${ }^{1}$ and $^{\text {G. Szabo }}{ }^{1}$
}

\begin{abstract}
Gut microbial translocation contributes to alcoholic hepatitis. Using a mouse model of alcoholic hepatitis, we investigated the effects of chronic alcohol plus binge and found increased abundance of Paneth cells and IL-17A in the proximal small intestine (PSI). Alcohol increased IL-17A production and pro-apoptotic signaling evidenced by Bax, Bim, caspase-3, and caspase-8 increases via endoplasmic reticulum (ER) stress indicated by C/EBP homologous protein (CHOP) upregulation; this was prevented by the ER stress inhibitor, 4-PBA, in isolated crypts in vitro and in vivo. Mechanistically, IL-17 augmented alcohol-induced ER stress in isolated crypts. In vivo IL-17A blocking antibody administration in alcohol-treated mice attenuated ER stress-mediated apoptosis and IL-18 induction and prevented alcohol-induced impairment of tight junctions in the PSI and LPS translocation to the liver. Acute-onchronic alcohol resulted in inflammasome activation, caspase-1 cleavage, and IL-18 production in the PSI. In vivo treatment with antibiotics or 4-PBA prevented CHOP upregulation and inflammasome activation. Our data suggest that alcohol upregulates innate immune mechanisms by increasing Paneth cell numbers and IL-17A release contributing to apoptosis amplification, inflammasome activation, and gut leakiness in the PSI. Binge alcohol-induced Paneth cell expansion, ER stress, and inflammasome activation in the PSI are modulated by the gut microbiome.
\end{abstract}

Mucosal Immunology (2019) 12:930-944; https://doi.org/10.1038/s41385-019-0170-4

\section{INTRODUCTION}

Alcohol-induced intestinal barrier dysfunction contributes to the development of alcoholic liver disease and alcoholic hepatitis. ${ }^{1}$ Previous studies have shown that chronic alcohol consumption changes the composition of the gut microbiome, results in inflammatory cytokine expression in the small bowel and disrupts the epithelial tight junction barrier that collectively lead to increased circulating levels of pathogen-associated molecular patterns (PAMPs) including bacterial lipopolysaccharide (LPS)., ${ }^{2,3}$ It has been shown that PAMPs and alcohol-induced damageassociated molecules (DAMPs) contribute to tissue inflammation in the liver and other organs. ${ }^{4}$

Paneth cells (PCs), immune-like epithelial cells of the crypts of Lieberkühn, are increasingly recognized as important regulators of intestinal homeostasis that shape gut microbial composition and restrain bacterial translocation., ${ }^{5,6}$ PCs produce antimicrobial peptides (lysozyme, cryptdins, phospholipase A [PLA2g2a]) and inflammatory cytokines that are readily induced and secreted in response to various microbial or inflammatory stimuli. Moreover, PC-derived interleukin (IL)-17 is an important regulator of the tight junction, ${ }^{7}$ and has been implicated in the pathogenesis of various organ injuries. ${ }^{8}$ PCs have a highly secretory phenotype with active endoplasmic reticulum (ER) function that renders them susceptible to perturbations in ER function, ${ }^{9}$ as occurs in other organs after chronic alcohol consumption. ${ }^{10}$
ER stress and activation of the unfolded protein response (UPR) have been identified both as physiologic and pathogenic factors in the healthy and diseased intestine. Accumulation of nascent or misfolded proteins in the ER leads to dissociation of the ER chaperone, Bip, from its binding partners, inositol-requiring enzyme 1 (IRE1), activating transcription factor 6 (ATF6), and PKR-like ER kinase (PERK), allowing them to initiate adaptive, proapoptotic, and pro-inflammatory signaling cascades. ${ }^{11}$ When ER stress is prolonged or cannot be resolved, the UPR signaling switches from pro-survival to pro-apoptotic, activating C/EBP homologous protein (CHOP) and growth arrest and DNA damageinducible gene 34 (GADD34) and transmitting pro-apoptotic signals from the ER to the mitochondria through pore-forming small proteins such as Bax, Bim, and Bid. ${ }^{12}$ Recently, ER stress has also been directly and indirectly associated with inflammatory signaling. Of particular interest, ER stress induced caspase-2 activation and mitochondrial damage have been shown to lead to the activation of the inflammasome, ${ }^{13}$ a multi-protein complex that acts through caspase-1, which cleaves and activates pro-IL-1 $\beta$ and pro-IL-18. ${ }^{14}$

Alcohol was found to induce ER stress in different tissues, ${ }^{10}$ however the role of UPR signaling and/or the contribution of PCs is yet to be evaluated in alcohol-induced changes in the intestinal mucosa. Here, we show that alcohol consumption results in intestinal, pro-apoptotic and pro-inflammatory signaling, and that PCs are susceptible to alcohol-related changes in the SI.

${ }^{1}$ Department of Medicine, University of Massachusetts Medical School, Worcester, MA 01605, USA

Correspondence: G Szabo (gyongyi.szabo@umassmed.edu)

These authors contributed equally: B. Gyongyosi, Y. Cho

Received: 16 March 2018 Revised: 5 April 2019 Accepted: 13 April 2019

Published online: 19 May 2019 


\section{RESULTS}

Alcohol induces cell death and inflammation in the proximal small intestine and leads to bacterial product translocation

Alcohol consumption leads to many physiologic changes throughout the body, beginning in the gastrointestinal tract. Using a model of chronic alcohol plus binge feeding in mice that results in features of early alcoholic hepatitis, ${ }^{15}$ we initially measured bacterial translocation from the intestine and found evidence of bacterial product translocation within the liver. Both bacterial $16 \mathrm{~S}$ ribosomal DNA (rDNA) and the bacterial wall component, lipopolysaccharide (LPS), were increased in the liver of alcoholfed mice compared with pair-fed animals (Fig. 1a, b). Since the liver is the first organ encountered by the portal circulation after the intestine, we sought to investigate the effects of alcohol on the small intestine. In the proximal small intestine (PSI), we observed that compared with pair-fed mice, alcohol-fed mice had significantly more cell death detected by measuring the extent of Terminal deoxynucleotidyl transferase dUTP Nick-End Labeling (TUNEL) (Fig. 1C, d). In addition, we measured the expression of multiple PSI proinflammatory cytokines including Tnfa, $\|6\| ,1 \beta$, $M c p 1, \| 123$, and $1 / 17 a$ and found that alcohol increased the mRNA expression of each of these cytokines (Fig. 1e). Together, these data suggest that alcohol induces significant changes in the PSI, including cell death and proinflammatory signaling, and these changes correlate with translocation of bacterial products from the intestinal lumen to the liver.

Alcohol increases the frequency of Paneth cells in the PSI and results in their degranulation

Paneth cells (PCs) are localized in the intestinal crypts of Lieberkühn and show increased abundance from the proximal to the distal segment of the $\mathrm{SI}$ in healthy intestines. ${ }^{16}$ We next investigated whether the distribution of PCs changed upon alcohol feeding in mice. We first measured PC frequency using PAS-staining (Fig. 2a) and immunohistochemistry for the PC marker, lysozyme, (Fig. 2b) in $\mathrm{SI}$ sections. We found a significant increase in the frequency of PCs in the PSI of alcohol-fed mice compared with control pair-fed mice (Fig. 2c, d). Remarkably, the alcohol-induced increase in PC frequency was restricted to the PSI and alcohol feeding did not change PC numbers in the distal SI (DSI) compared with controls. We also found that alcohol feeding resulted in crypt degranulation, indicated by PAS-positive material in the lumen of crypts, suggesting that alcohol exposure promotes release of antimicrobial substances from the PCs (Fig. 2a inserts and e).

PCs play a role in anti-microbial defense and in intestinal selfrenewal. ${ }^{17}$ Thus, we tested expression of genes implicated in PC differentiation and function and found increased mRNA expression of differentiation markers, Sox9 and Igfbp $4{ }^{18}$ Spdef and Math $1{ }^{19}$ and Lgr5, while the inhibitory KIf $4^{20}$ was decreased in the PSI of alcohol-fed mice compared with pair-fed mice (Fig. 2f). Although these genes could be expressed by multiple cell types, these findings are consistent with the increased numbers of PCs in the PSI after alcohol feeding. The expression of antimicrobial peptide genes (Cryptdins; defensins Defcr1, Defcr4, and Defa-rs1; PLA2g2a; lysozymes $L y z 1$ and $L y z 2$; and Muc2) was also significantly higher in the PSI of alcohol-fed compared with control diet-fed mice (Fig. 2g). Indeed, an acute binge increased Cryptdins expression after both 4 $\mathrm{h}$ and $9 \mathrm{~h}$, while mice treated with chronic alcohol and no binge did not show an increase in expression (Suppl. Fig. 2).

Alcohol induces IL-17 in the PSI and in isolated small intestinal crypts

Recent studies support that IL-17 contributes to gut homeostasis, $^{21}$ however the role of IL-17 in alcohol-induced gut permeability is unknown. Immunoblot and immunohistochemistry analyses revealed increased IL-17A levels in the PSI of alcohol-fed compared with pair-fed mice (Fig. 3a-d). The IL-17A staining was localized to the bottom of the crypts identical to the localization of
PCs (Fig. 3a). However, IL-17 could also be released by a subset of $T$ cells, the $T$ helper (Th)-17 cells. Therefore, we isolated resident intestinal immune cells of the PSI lamina propria and quantified Th17 cells by flow cytometry. We found no differences in the number of Th17 cells between alcohol-fed and pair-fed mice (Fig. 3e). These results suggest that PCs are likely the source of alcohol-induced intestinal IL-17A increase.

Having observed PC expansion and degranulation in alcohol-fed mice, we aimed to explore mechanisms by which ethanol might regulate PC function. Therefore, we isolated crypts that are enriched in PCs from wild-type mice and exposed them to different doses of ethanol ex vivo. Ethanol exposure of these isolated PSI crypts resulted in a significant increase in IL-17A release that correlated with the in vivo observation of increased IL-17A in PCs in the SI in alcohol-fed mice. The ex vivo ethanol-induced IL-17A release was time-dependent and dose-dependent, beginning at $2 \mathrm{~h}$ and peaking at $6 \mathrm{~h}$ (Fig. 3f). The IL-17A increase detected in the supernatants was due to secretion from viable crypts and not a consequence of ethanol-induced cell death because there was no increase in lactate dehydrogenase (LDH) cytotoxicity assay in EtOH-treated crypts compared with controls (Suppl. Fig. 1)

Alcohol-induced IL-17A release is mediated by ER stress PCs are highly secretory and, consequently, susceptible to ER stress. ${ }^{9}$ We therefore hypothesized that alcohol-induced ER stress might be involved in IL-17 release. To test this hypothesis, we measured the effect of 4-PBA (a FDA-approved ER stress inhibitor) on ethanol-induced IL17A release. ${ }^{22}$ We found that administration of 4-PBA prevented alcohol-induced IL17A release, in a dosedependent manner (Fig. 4a). Conversely, IL-17A release was induced by treatment with the ER stress inducer, thapsigargin, in a dose-dependent manner with increasing IL-17A detected in crypt supernatants following $0.3,1$, and $3 \mu \mathrm{M}$ thapsigargin (Fig. $4 \mathrm{~b}$ ). These results indicate that alcohol induces IL-17A release in $\mathrm{SI}$ crypts via an ER stress-dependent mechanism.

Next, we sought to investigate the role of IL-17A on cell signaling pathways in the SI. SI crypts treated with recombinant IL17A ex vivo had increased cleavage of PARP that was not affected further by alcohol exposure (Fig. 4c, d). Administration of an IL-17A blocking antibody in vivo abrogated PARP cleavage following acute-on-chronic alcohol exposure. Together, these data suggest an IL-17-mediated process of PSI apoptosis. Interestingly, in vivo administration of the IL-17A blocking antibody also showed a trend of reduction in the cleavage and activation of caspase-8, while control antibody had no effect (Fig. 4e, f).

Acute-on-chronic alcohol administration induces pro-apoptotic signaling and ER stress in the PSI

ER stress and activation of the unfolded protein response (UPR) are key features of secretory cells, such as Paneth cells. ${ }^{23}$ Given the important connections between IL-17A and ER stress and that prolonged ER stress initiates cell death signaling by relaying stress signals from the ER to the mitochondria, we next investigated protein levels of the pro-apoptotic molecules, Bax, Bim, and Bid. These proteins were reported to be central in ER stress-induced apoptosis pathways acting downstream of CHOP signaling. ${ }^{12}$ We found that 10-day alcohol feeding alone (10 day EtOH no binge) did not significantly increase expression of the pro-apoptotic molecules Bax, Bim, and Bid (Fig. 5a, b). However, the acute binge on chronic alcohol diet significantly upregulated the protein levels of Bax, Bim, and Bid in the PSI both 4 and $9 \mathrm{~h}$ after the binge (Fig. 5a, b). These molecules are responsible for mitochondrial outer membrane permeabilization leading to downstream caspase activation. In line with this, we found increased cleavage of PARP (Fig. 5a, b), a well-characterized marker of caspase activation.

ER stress is compensated by activation of the unfolded protein response, a homeostatic signaling mechanism that orchestrates recovery of ER function and upon failure to adapt to ER stress, 


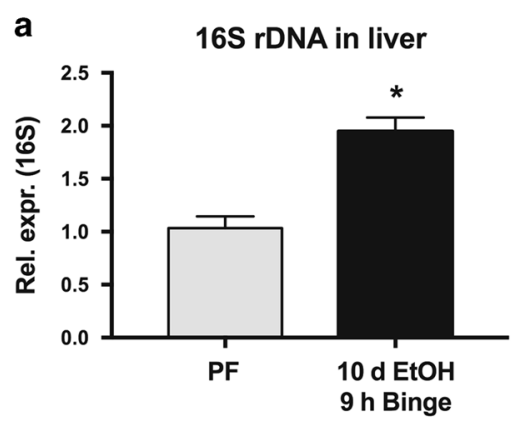

C

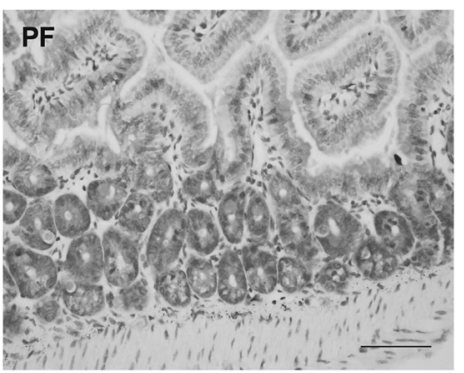

e
TUNEL in PSI

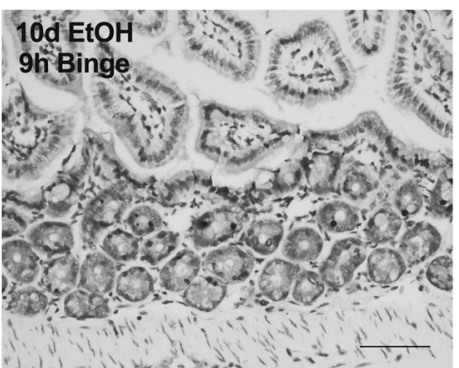

Cytokine expression in PSI

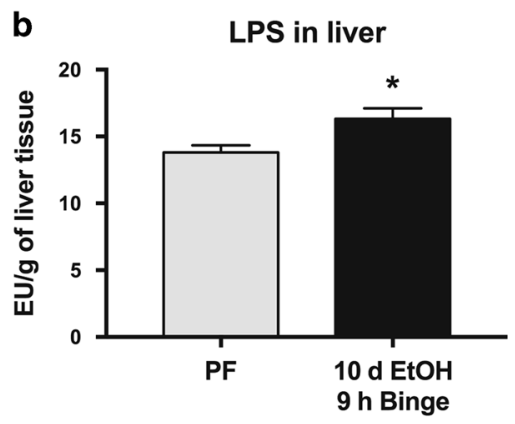

d
TUNEL (+) cells in PSI

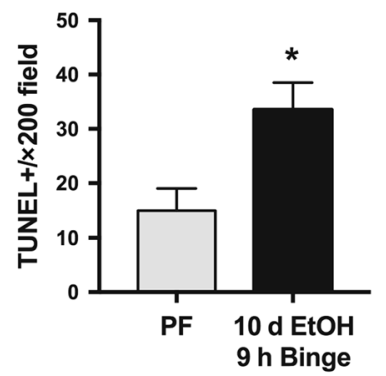

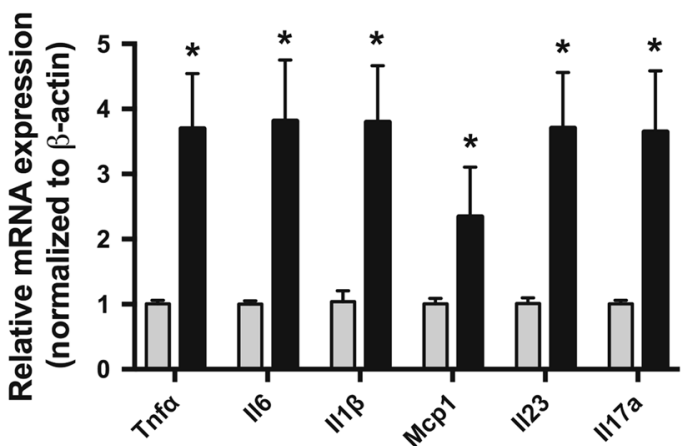

$\square$ PF
$10 \mathrm{~d} \mathrm{EtOH}$
$9 \mathrm{~h}$ Binge

Fig. 1 Alcohol induces cell death and inflammation in the proximal small intestine and leads to bacterial product translocation. a Bacterial product translocation to the liver was detected by GPCR of bacterial 16s rDNA and (b) by chromogenic endotoxin quantification of liver LPS levels after calorie-controlled pair-fed diet (PF) or 10 days of $5 \%$ alcohol in liquid diet plus binge alcohol (10 day EtOH $9 \mathrm{~h}$ binge) in mice. c-d Cell death was measured in the proximal small intestine (PSI) using Terminal deoxynucleotidyl transferase dUTP Nick-End Labeling (TUNEL) staining. e mRNA expression levels of inflammatory cytokines and chemokines, Tnfa, II-6, II1 $\beta, M c p 1,\|23\|$,$17 a , were measured by$ qPCR. $n=5-7$ mice/group; ${ }^{*} p<0.05$

results in apoptosis. We found a significant and selective upregulation of p-eIF2a, a marker of the PERK arm of ER stress, and CHOP protein expression in the PSI of mice after 10 days of alcohol feeding compared with controls (Fig. 5a, b). Acute binge in the chronic alcohol-fed mice resulted in a further significant increase in CHOP, phosphorylation of elF2a and also increased GADD34 (a downstream target of CHOP) levels at 4 and $9 \mathrm{~h}$ after the acute binge (Fig. 5a, b). Interestingly, these alcohol-induced changes were not observed in the DSI (Suppl. Fig. 3). These data suggest that chronic alcohol induces $\mathrm{CHOP}$, and an additional acute alcohol binge augments CHOP expression and downstream pro-apoptotic events, including translational block (phosphorylation of elF2a) and GADD34 induction in the PSI.

\section{Alcohol increases caspase expression and activation through ER} stress

Next, we measured the expression and activation of caspases involved in apoptosis pathways. We detected cleavage of the executioner caspase, caspase-3, indicative of ongoing apoptotic signaling at 4 and $9 \mathrm{~h}$ after binge in chronically alcohol-fed mice (Fig. 5c, d). We also found increased levels of total and cleaved Bid (tBid) at $9 \mathrm{~h}$ after the acute alcohol binge (Fig. 5a, b). Bid is a known caspase- 8 substrate in the extrinsic apoptotic pathway, and has been described as a mediator of the caspase-2 induced inflammasome activation upon ER stress. ${ }^{13}$ Therefore, we measured these two caspases and found a significant induction of both caspase- 8 and caspase- 2 protein levels as early as $4 \mathrm{~h}$ after the acute binge (Fig. 5c, d), suggesting that acute-on-chronic alcohol activates both extrinsic and intrinsic pro-apoptotic mechanisms in the PSI.

In order to determine the role of ER stress in small intestinal caspase activation, we treated mice in vivo with the ER stress inhibitor, 4-PBA. Acute-on-chronic alcohol administration led to the induction of CHOP expression (Fig. 5a, b), as well as caspase-8 (Fig. 5c, d). 4-PBA administration inhibited ER stress, determined by decreased CHOP expression (Fig. 5e, f). Interestingly, 4-PBA also abrogated the alcohol-induced increase in activation of caspase 8 (Fig. 5e, f), indicating that alcohol-induced caspase activation is due to the induction of ER stress. 
a

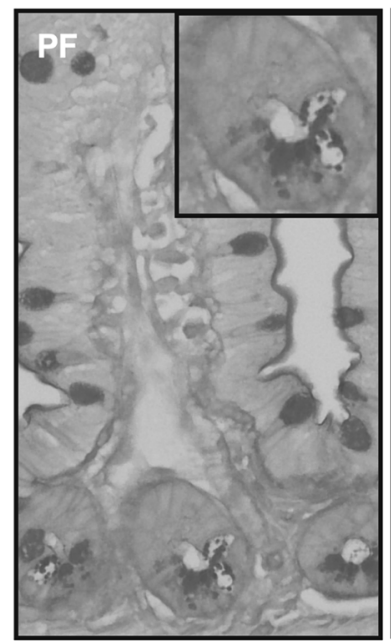

Paneth cells in PSI (PAS)

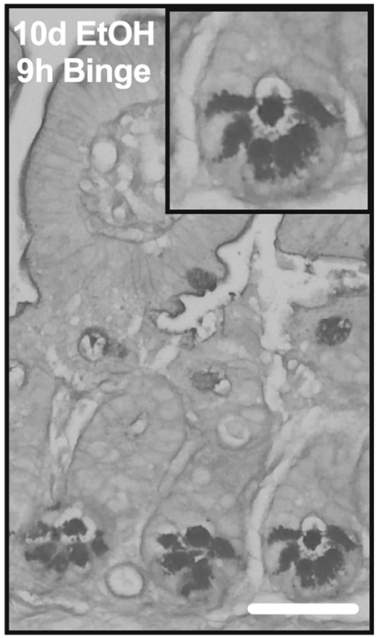

b

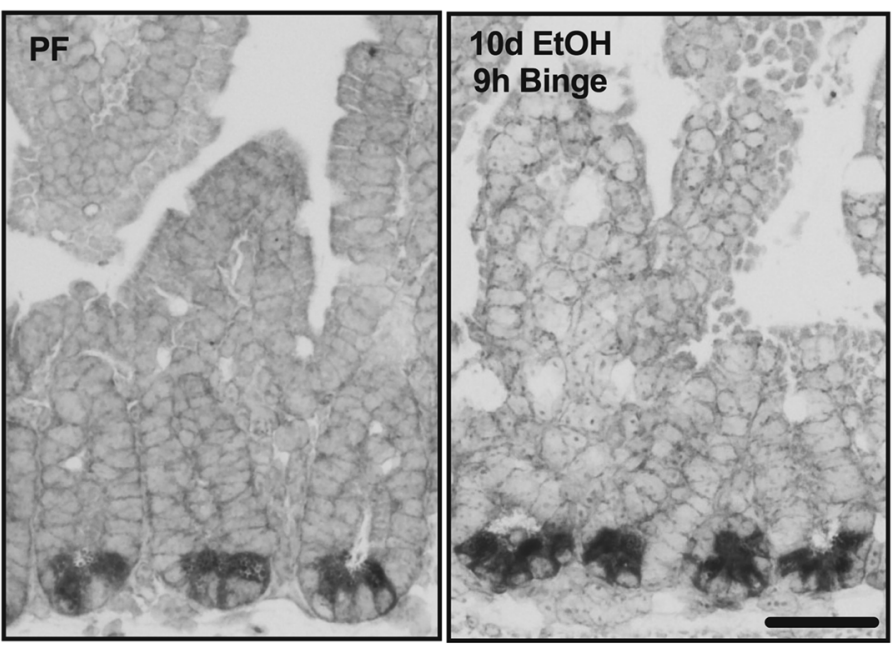

C

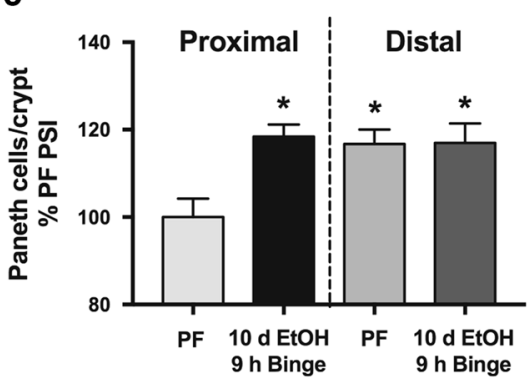

d

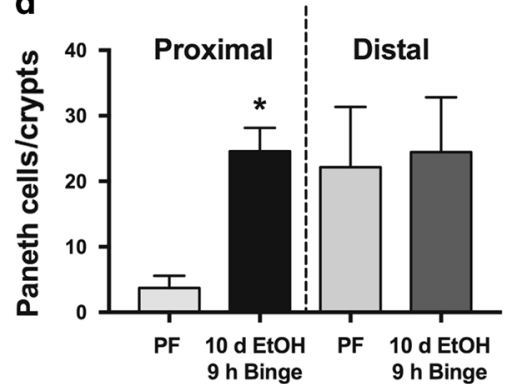

e

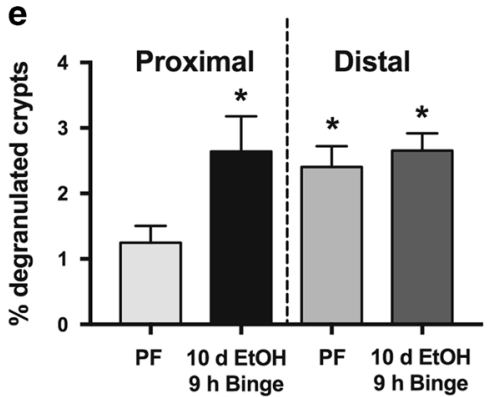

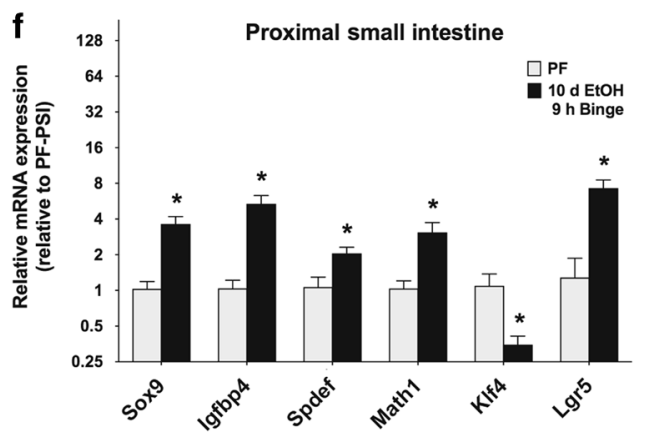

Distal small intestine

$$
\square \mathrm{PF}
$$

$\square 10 \mathrm{~d}$ EtOH $\mathrm{h}$ Binge

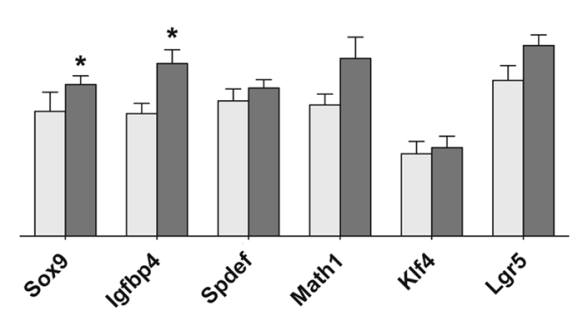

g
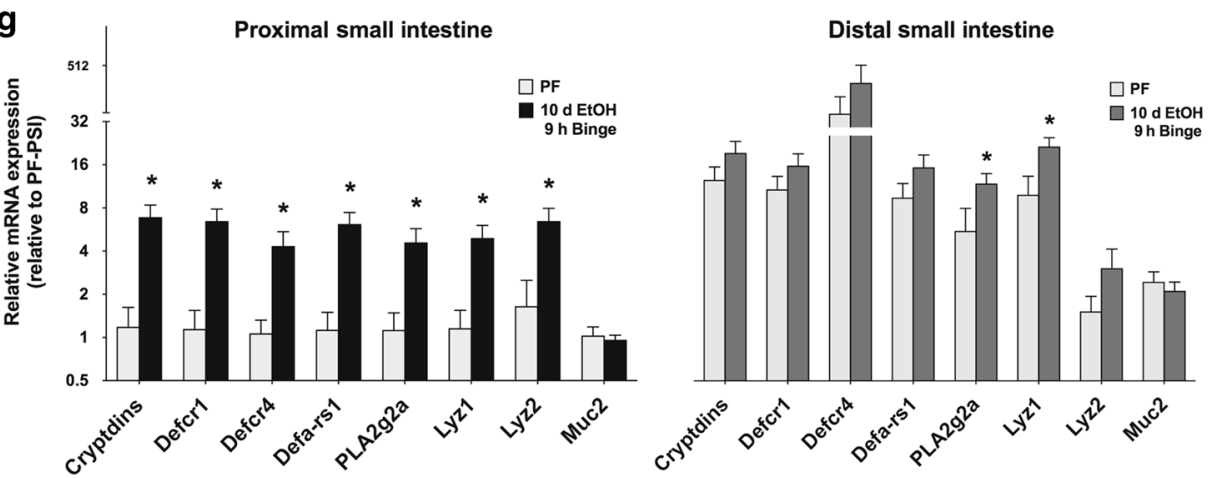

Fig. 2 Alcohol increases the frequency of Paneth cells in the proximal small intestine and results in their degranulation. a Representative images and (c) quantification of PAS-stained proximal small intestinal sections. Inserts show individual crypts. b Representative images and (d) quantification of lysozyme immunohistochemistry (IHC) in PCs from pair-fed (PF) or 10 day EtOH $9 \mathrm{~h}$ binge alcohol-fed mice. e Crypt degranulation (highlighted by magnifications of (a) where PAS-positive material is observed in the crypt lumen in EtOH) was measured in the PSI and the DSI after 10 day EtOH $9 \mathrm{~h}$ binge alcohol exposure. $\mathbf{f}$ Expression of differentiation and stem markers, including Sox9, Igfbp4, Spdef, Math1, KIf4 and Lgr5, were measured by qPCR in the PSI and DSI in alcohol-fed and pair-fed mice. g Expression of antimicrobial peptide genes, including Cryptdins; defensins Defcr1, Defcr4, and Defa-rs1; PLA2g2a; lysozymes Lyz1 and Lyz2; and Muc2, were also measured by qPCR. $n=5-7$ mice/group; * $p<0.05$ 
a

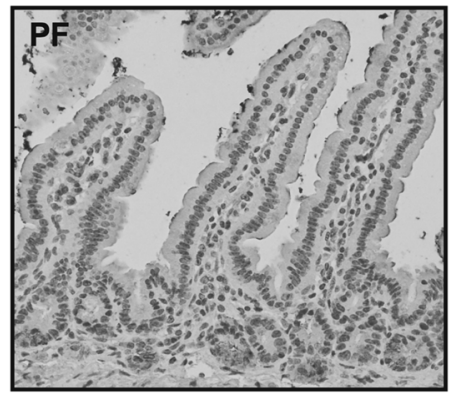

IL-17A in PSI (IHC)

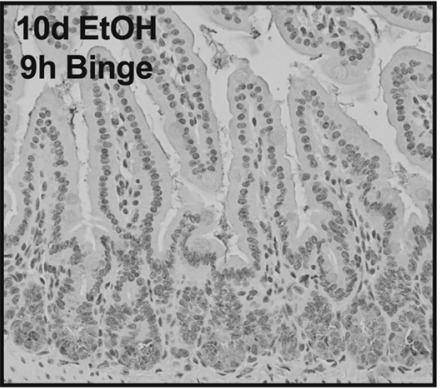

b

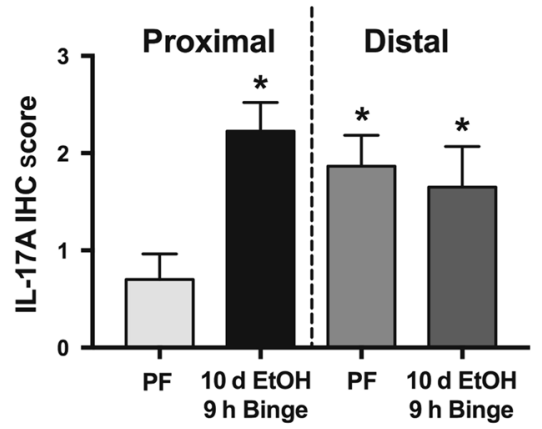

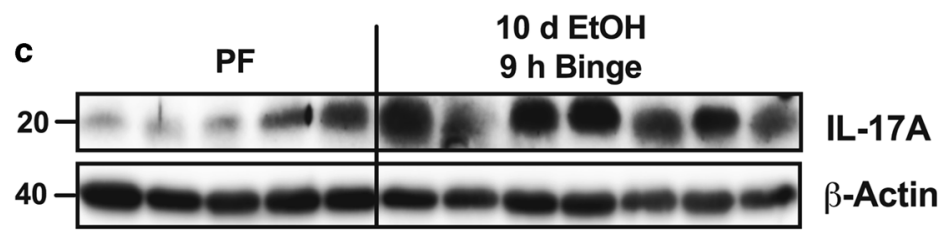

d

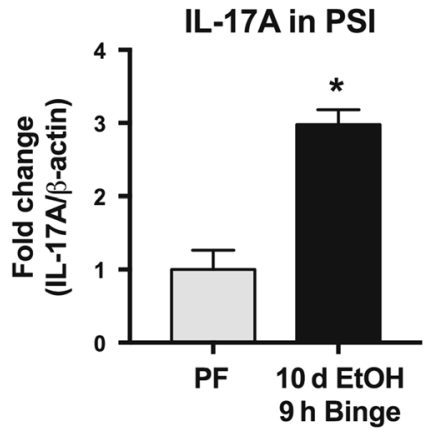

e

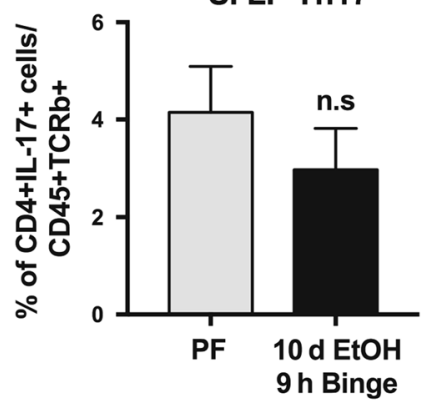

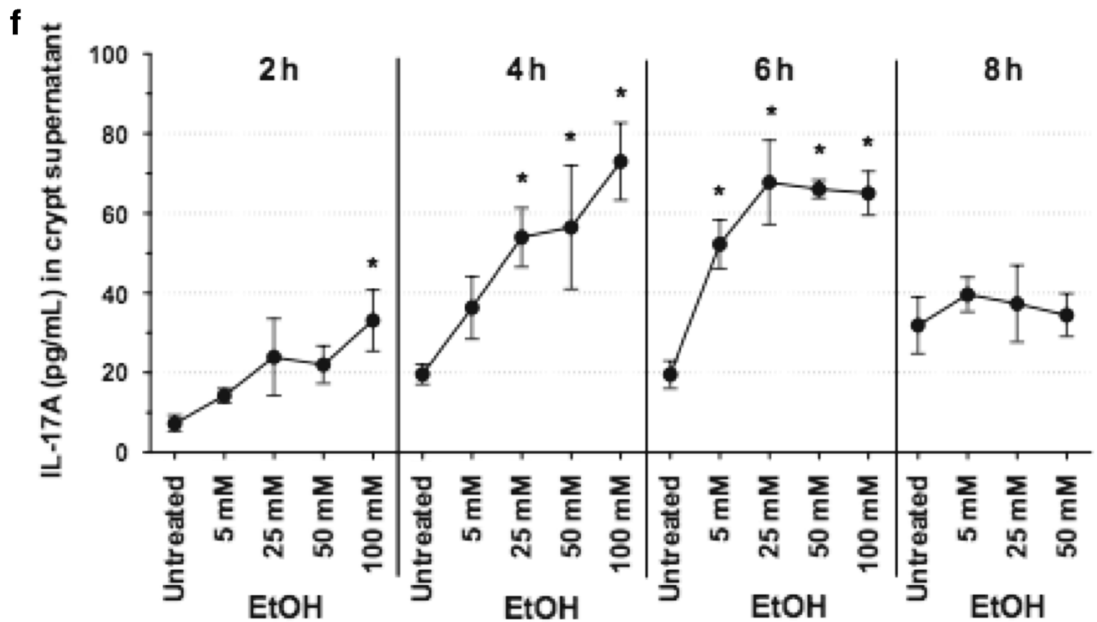

Fig. 3 Alcohol induces IL17A expression in Paneth cells but not Th17 cells in the proximal small intestine. a Representative images and (b) quantification of IL-17A immunohistochemistry (IHC) in 10 day EtOH $9 \mathrm{~h}$ binge alcohol-fed and pair-fed (PF) mice. c IL-17A from whole PSI protein was also quantified (d) by Western blot. e Flow cytometry of small intestinal lamina propria (SI LP) detected Th17 cells, defined as $\mathrm{CD} 45+, \mathrm{TCRb}+, \mathrm{CD} 4+, \mathrm{IL}-17+$ live single cells. $\mathrm{f}$ Isolated small intestinal crypts were stimulated ex vivo with various concentrations of ethanol $(0,4,25,50$, and $100 \mathrm{mM})$ for $2,4,6$, or $8 \mathrm{~h}$ and IL-17A was measured from the supernatants by ELISA. $n=5-7$ mice/group; ${ }^{*} p<0.05$

Alcohol-mediated increase of CHOP is localized to Paneth cells in PSI crypts

To characterize the cell type most affected by alcohol, we next isolated epithelial cell (containing PCs) and immune cell fractions (containing intraepithelial and lamina propria leukocytes) from the PSI. Western blot analysis revealed a significant upregulation of both the ER stress proteins CHOP and Bax in the epithelial cell fraction (Fig. 6a, b, upper panels), but not in the intraepithelial (IEL) or lamina propria (LPL) immune cell fractions (Fig. 6a, b, lower panels), suggesting that alcohol primarily affects epithelial cells. Immunofluorescent (IF) staining revealed increased CHOP fluorescent intensity in PCs of the crypts in the PSI of alcohol-fed mice compared with controls (Fig. 6c, d). Finally, we isolated crypts from the PSI of pair-fed or alcohol-fed mice in order to differentiate the protein expression localized in this particular epithelial cell layer as the epithelial cell isolation includes both crypts (containing the 
a

IL-17A in crypts Sup.
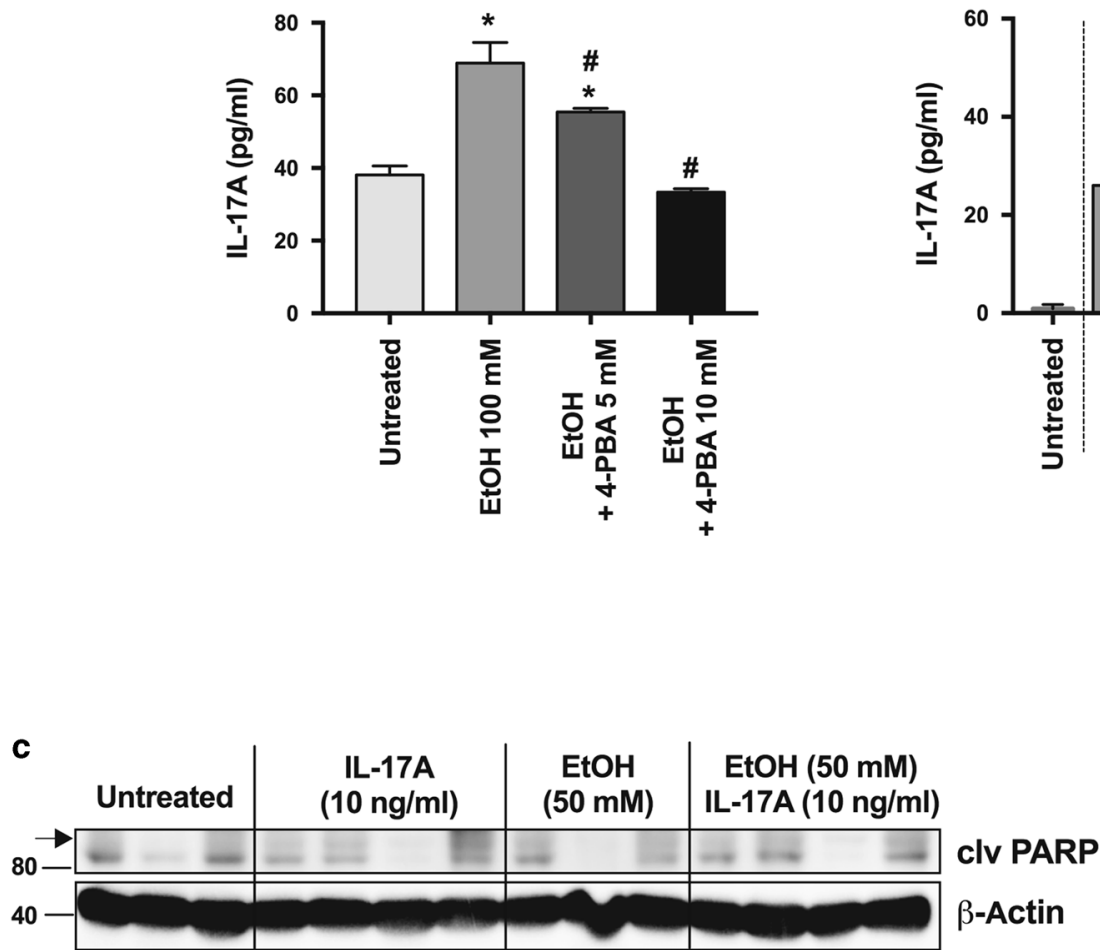

e

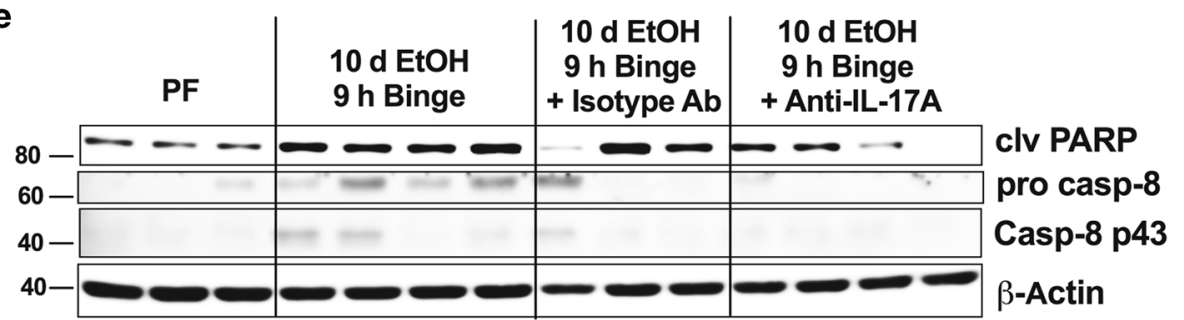

$\mathbf{f}$

Cleaved PARP in PSI

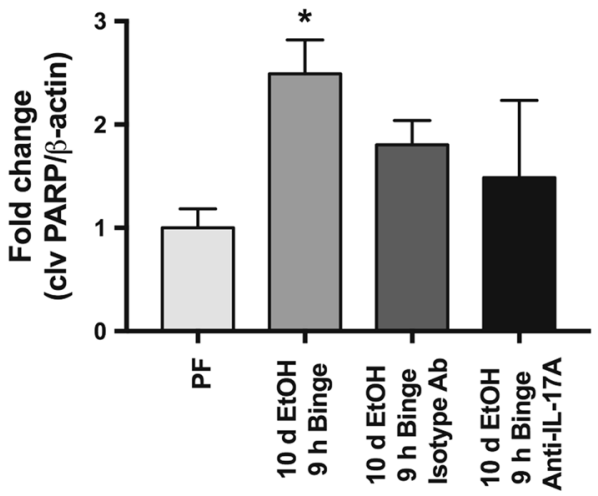

PCs) and villus epithelial cells. In the isolated crypts, we again detected an increase in ER stress proteins Bax and Bim in alcoholfed compared with pair-fed mice (Fig. 6e, f). These results identified that in vivo alcohol increases CHOP expression in PCs in the crypts of the PSI. b

IL-17A in crypts Sup.

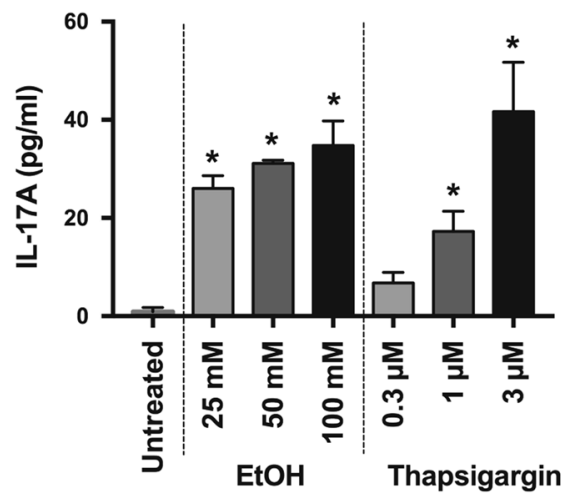

d

Cleaved PARP in isolated crypts (PSI)

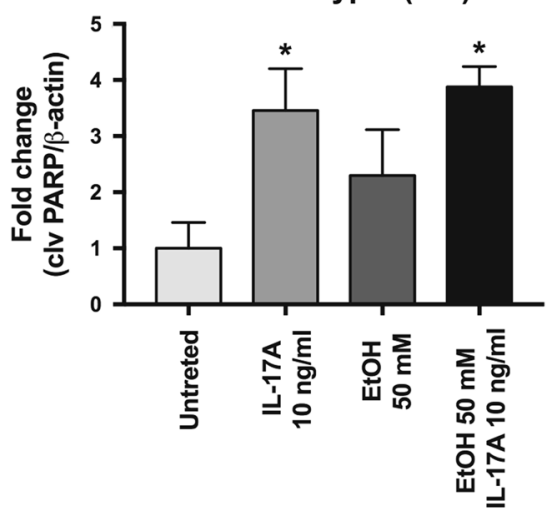

g

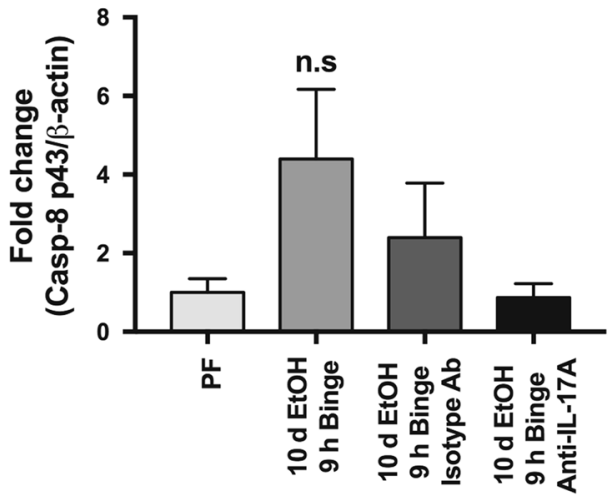

Alcohol-reduced intestinal barrier integrity is restored by anti-IL-17 treatment in vivo

To assess the role of IL-17 in alcohol-induced intestinal barrier dysfunction, we used an IL-17 blocking antibody in vivo and found that IL-17 blocking prevented the alcohol-induced increase in liver 
Fig. 4 Ethanol-induced IL-17A release is augmented by ER stress. a Isolated small intestinal crypts were treated with ethanol (100 mM) to induce IL-17A release and some crypts were also treated with 5 or $10 \mathrm{mM}$ of the ER stress inhibitor 4-phenylbutiric acid (4-PBA). Released IL-17A was measured by ELISA from the crypt supernatants. b IL17A release was measured by ELISA performed on isolated crypts treated with the ER stress inducer thapsigargin $(0.3,1$, and $3 \mu \mathrm{M})$ or ethanol $(25,50$, and $100 \mathrm{mM})$. c Cleaved (clv) PARP expression was measured by Western blot and quantified (d) from isolated crypts treated ex vivo with $10 \mathrm{ng} / \mathrm{ml}$ of recombinant IL-17A, $50 \mathrm{mM}$ EtOH or IL-17A and EtOH. e Cleaved PARP, pro-caspase 8 and cleaved caspase 8 p43 subunit from the PSI of pair-fed (PF) or alcohol-fed mice treated or not with a control isotype antibody or IL17A blocking antibody were measured by Western blot and quantified (f). For $\mathbf{a}-\mathbf{b}, n=3$ mice/treatment group; $p<0.05$ ${ }^{*}$ vs. untreated, \# vs. EtOH $100 \mathrm{mM}$. For $\mathbf{d}-\mathbf{e}, n=3-4, p<0.05 *$ vs. Untreated. For $\mathbf{f}-\mathbf{g}, n=3-4$ mice/group; $p<0.05 *$ vs. PF

LPS levels (Fig. 7a), suggesting a connection between IL-17 and the integrity of the intestinal barrier. Therefore, we next evaluated tight junction protein expression in the PSI. Although changes in barrier integrity are likely to occur as a continuum across time of exposure to alcohol, our acute-on-chronic alcohol feeding model dramatically reduced the immunofluoresence of zonula occludens-1 (ZO-1), an important component of the PSI tight junction structure (Fig. 7b). Treatment with IL-17 blocking antibody recovered expression of ZO-1, while ZO-1 staining was unaffected by the isotype antibody (Fig. 7b). Anti-IL-17 also prevented the alcohol-induced decrease in occludin dimer protein expression in the PSI (Fig. 7c, d). We found that IL-17 blocking resulted in a trend of upregulation in the mRNA expression of claudin-1, claudin-5, and claudin-15, as well as occludin and ZO-1 (Fig. 7e). Interestingly, the acute-on-chronic alcohol showed no reduction at the mRNA levels of these tight junction proteins that is likely due to the binge alcohol effect. ${ }^{2}$

Inflammasome activation by acute alcohol binge in the small intestine is attenuated by in vivo inhibition of ER stress

ER stress is known to induce inflammation and PCs are important for the initiation of intestinal inflammation. ${ }^{24}$ Therefore, we investigated activation of the central sensor and orchestrator of inflammatory signaling, the inflammasome, in the acute-onchronic alcohol feeding. We found activation of caspase- 1 was significantly increased in the PSI of acute-on-chronic mice compared with PF and chronic alcohol alone (Fig. 8a, b). Inflammasome activation was indicated by cleavage of the inflammasome effector, caspase-1 (p10), and its downstream target, IL-18 (18 kDa cleaved form) that were increased in mice after acute binge alcohol (Fig. 8a, b).

To investigate whether ER stress could play a role in inflammasome activation, we administered the ER stress inhibitor, 4-PBA, in the liquid diet during alcohol feeding. We found that alcohol-related CHOP expression was attenuated in 4-PBA-treated mice indicating effective ER stress inhibition (Fig. 8c, d). Importantly, in vivo administration of 4-PBA in alcohol-fed mice resulted in decreased IL-18 cleavage indicating reduced inflammasome activation (Fig. 8c, d). In addition, 4-PBA treatment significantly reduced the cleavage of PARP (Fig. 8e, f). These data suggested that alcohol-induced ER stress contributes to inflammasome activation in the PSI. Because we had previously observed that induction of ER stress resulted in the release of IL-17 from PSI crypts (Fig. 4) and that ER stress contributes to inflammasome activation (Fig. 8c, d), we next measured the role of IL-17 in inflammasome activation and found that the induction of active IL-18 by alcohol is inhibited when mice were treated with an IL-17 blocking antibody (Fig. 8g, h).

Alcohol-induced CHOP induction, IL-18 activation, and increase in PC number are mediated by the gut microbiota load

Since inflammatory signals from the gut microbiome contribute to the pathogenesis of alcohol-induced organ damage, we investigated whether gut decontamination might affect the alcoholinduced changes in the PSI. We used a combination of antibiotics (ampicillin, neomycin, vancomycin and metronidazole) administered before and simultaneously with the alcohol or pair-fed diet.
Successful reduction in gut bacterial load was indicated by enlarged cecum and log-reduction of both aerobic and anaerobic colony forming units (Suppl. Fig. 4). We found that administration of antibiotics significantly reduced the alcohol-induced increase in CHOP and IL-18 cleavage in the SI (Fig. 9a, b). Moreover, antibiotics prevented the alcohol-induced increase in PC numbers measured by immunohistochemistry for lysozyme expression (Fig. 9c, d). These results suggest that signals from the gut microbiome are significant contributors to the development of alcohol-induced PSI pathology and PC functions (Fig. 10).

\section{DISCUSSION}

The biological effects of alcohol consumption on the Gl tract are not limited only to intestinal functions, but extend beyond the $\mathrm{Gl}$ tract due to alcohol-related changes in gut permeability. It has been shown that increased levels of LPS in the portal and systemic circulation contribute to the end-organ effects of alcohol in the liver, lung, and brain. ${ }^{4,25}$ Previous studies demonstrated disruption of tight junctions in animal models of alcoholic liver disease, as well as in human alcoholics. ${ }^{26}$ Furthermore, previous studies demonstrated pro-inflammatory pathway activation and increased levels of pro-inflammatory cytokines in the small bowel after chronic alcohol administration in mice. ${ }^{2}$ In this study, we report that acute-on-chronic alcohol, which induces early alcoholic hepatitis in the liver, ${ }^{3}$ modulates small intestinal homeostasis by increasing the numbers of Paneth cells and IL-17 production in the PSI. Mechanistically, we show that alcohol induces IL-17A production via ER stress and results in upregulation of the elF2a and CHOP arm of UPR by chronic alcohol that was further augmented by additional acute alcohol binge in the PSI. We showed acute-on-chronic alcohol induced pro-apoptotic pathways in the PSI and that IL-17 itself induces ER stress in isolated crypts. In vivo, IL-17 blocking antibody prevented the alcoholinduced gut permeability and improved gut barrier functions via normalizing ZO-1 and occludin expression. We also found that alcohol induced ER stress, pro-inflammatory genes upregulation, inflammasome activation and IL-18 production after acute-onchronic alcohol feeding in mice. Finally, decontamination of the gut with antibiotics prevented the alcohol-induced increase in Paneth cells numbers, CHOP upregulation and inflammasome activation in the PSI. Gut decontamination has also been shown to attenuate alcohol-induced liver inflammation ${ }^{3}$ and the data presented here provides further mechanistic evidence for this important gut-liver interplay.

The cells of the intestine serve a multitude of functions including the primary role of separating the contents of the intestine from the remainder of the body. Here, we show that acute-on-chronic alcohol exposure reduces the PSI gut barrier integrity leading to bacterial product translocation, although other regions of the intestinal tract may also have altered barrier integrity with increased translocation and these regions are an area of opportunity for future study. Reduced PSI integrity is associated with an increase in cell death in the PSI and an increase in pro-inflammatory cytokine expression. Paneth cells are the main contributors in the intestinal mucosal barrier by secreting large amounts of antimicrobial and inflammation-associated proteins. ${ }^{5-7,26}$ In the normal GI tract, the 
a

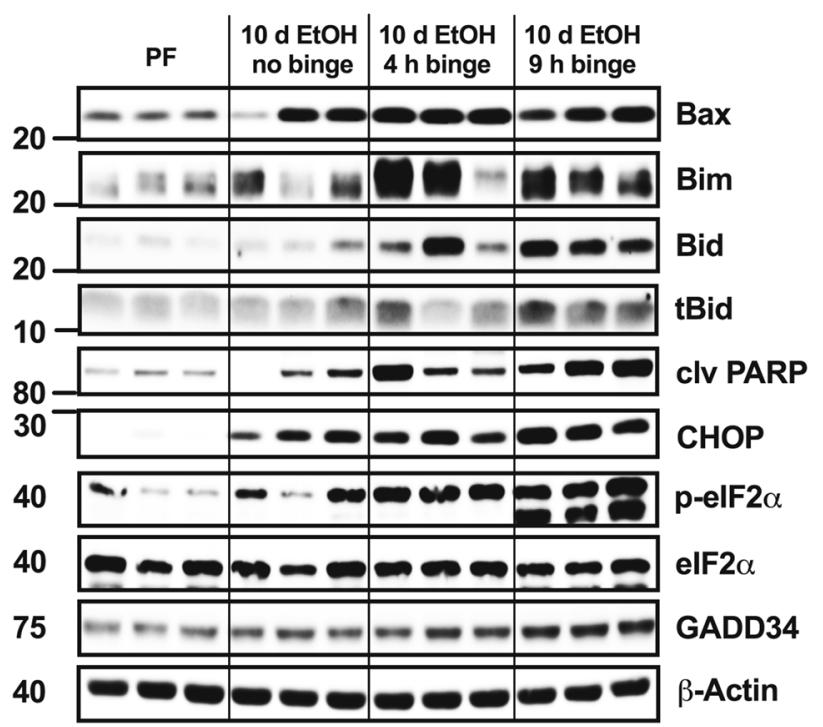

$\square$ PF

$\square 10 \mathrm{~d}$ EtOH no binge $10 \mathrm{~d} \mathrm{EtOH} 4 \mathrm{~h}$ binge $10 \mathrm{~d}$ EtOH 9 h binge

C

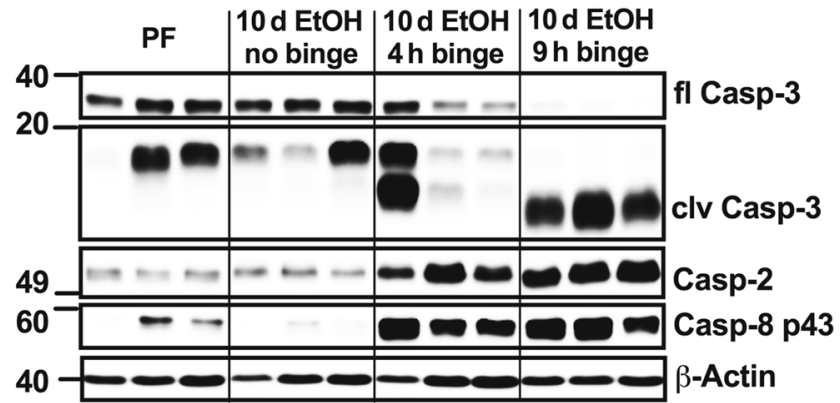

e

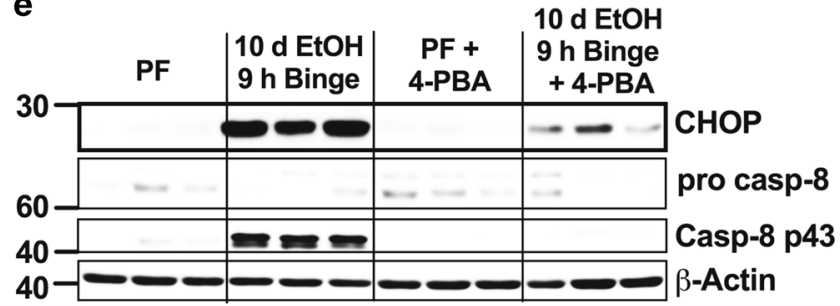

b

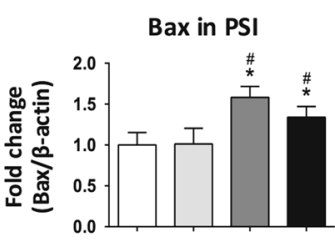

Bid in PSI

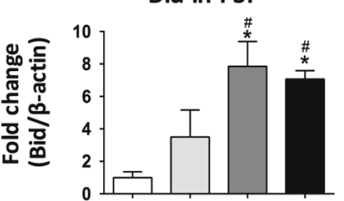

clvPARP in PSI
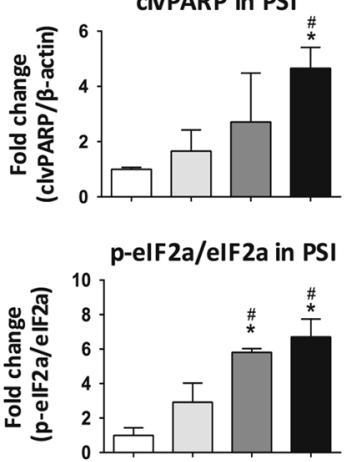

d
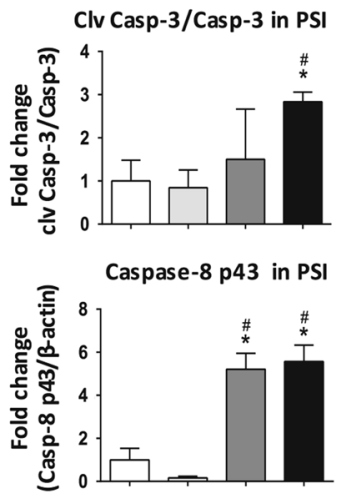

$\mathbf{f}$

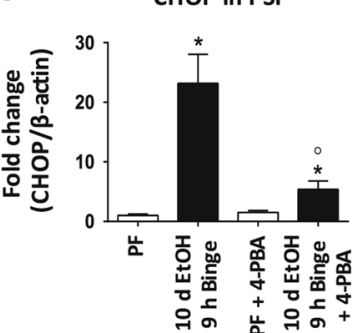

Bim in PSI

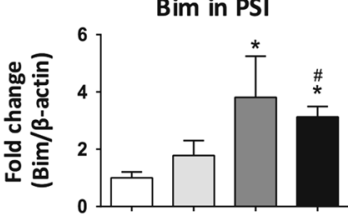

tBid in PSI

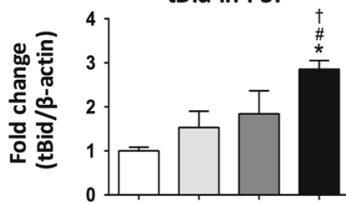

CHOP in PSI
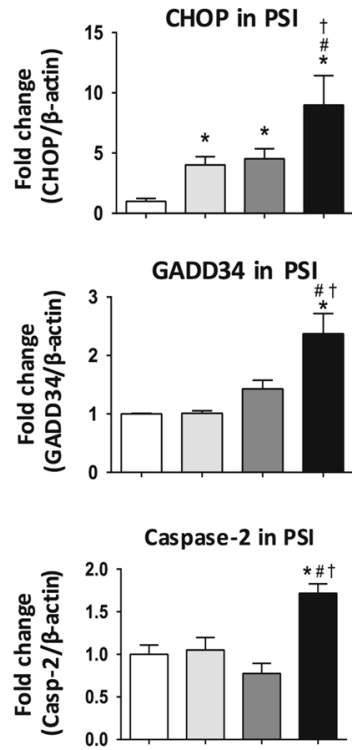

$\square \mathrm{PF}$

$\square 10 \mathrm{~d} \mathrm{EtOH}$ no binge

$10 \mathrm{~d}$ EtOH $4 \mathrm{~h}$ binge

$10 \mathrm{~d}$ EtOH $9 \mathrm{~h}$ binge

Fig. 5 Alcohol administration induces pro-apoptotic signaling and ER stress in the proximal small intestine. a Pro-apoptotic proteins Bax, Bim, Bid, truncated Bid (tBid) and cleaved (clv) PARP, as well as ER stress related proteins CHOP, p-elF2 $\alpha$, elF2 $\alpha$, GADD34 full length (fl) were measured by Western blot and quantified (b) from PSI tissue of pair-fed (PF) mice, as well as mice fed a 10-day chronic alcohol, a 10 day EtOH plus $4 \mathrm{~h}$ binge or a 10 day EtOH $9 \mathrm{~h}$ binge diet. c Caspase-3 (fl), cleaved (clv) caspase-3, caspase-2, and caspase-8 p43 were measured from the same PSI samples by Western blot and quantified (d). e Mice received either calorie matched diet (PF) or 10 day EtOH $9 \mathrm{~h}$ binge and some mice from each group also received in vivo administration of the ER stress inhibitor 4-phenylbutiric acid (4-PBA). Expression in the PSI of CHOP, pro-caspase- 8 and clv caspase-8 p43 were measured by Western blot and quantified (f). For A-D, $n=5-7$ mice/group; $p<0.05 *$ vs. pair-fed (PF), \# vs. 10 day EtOH no binge, + vs. 10 day EtOH $4 \mathrm{~h}$ binge. For e-f, $n=5-7$ mice/group; $p<0.05$ * vs. pair-fed, \# vs. 10 day EtOH no binge, $\dagger$ vs. 10 day EtOH $4 \mathrm{~h}$ binge, ${ }^{\circ}$ vs. 10 day EtOH $9 \mathrm{~h}$ binge 


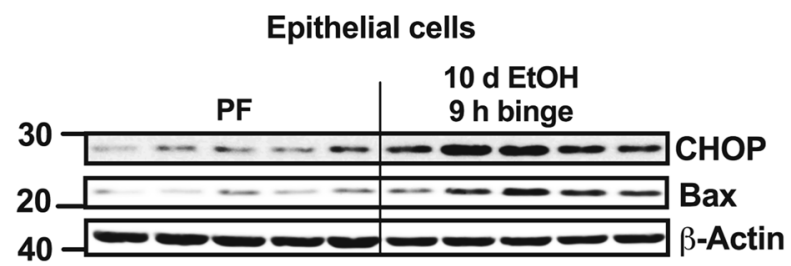

Immune cells
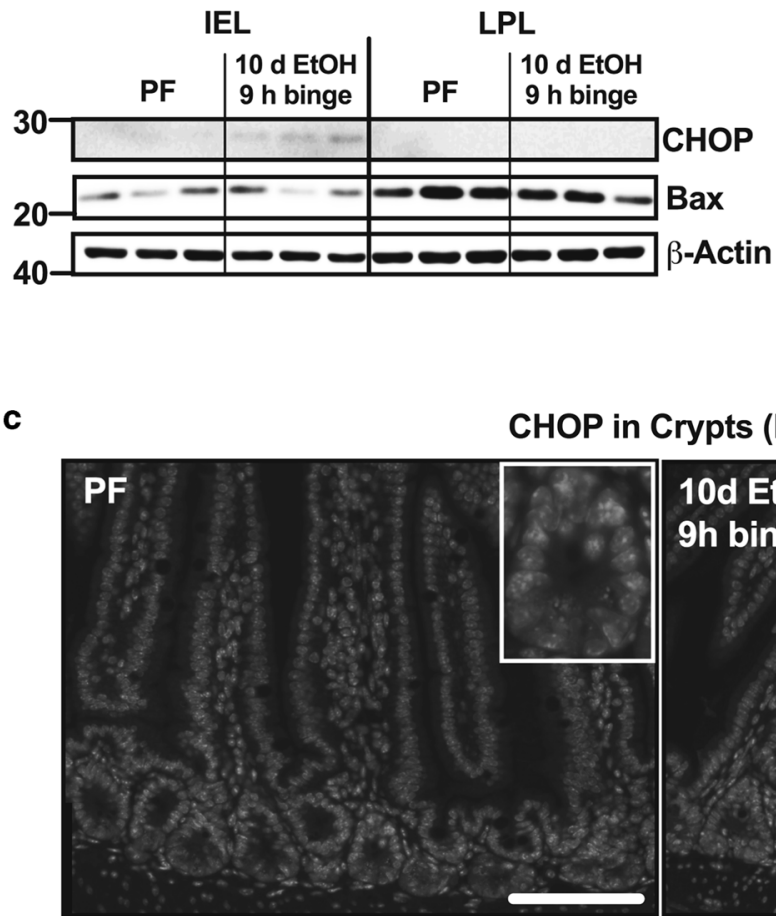

CHOP in Crypts (IF)

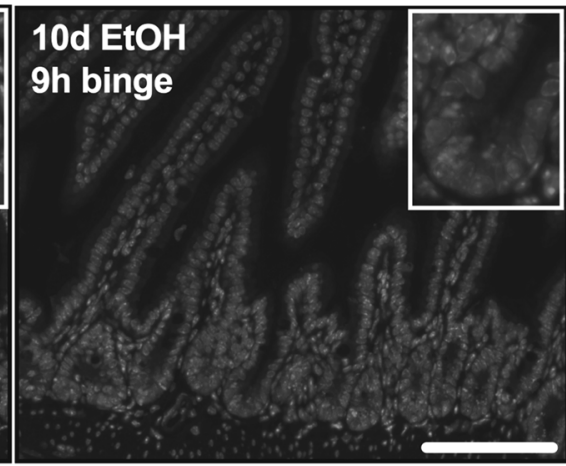

e

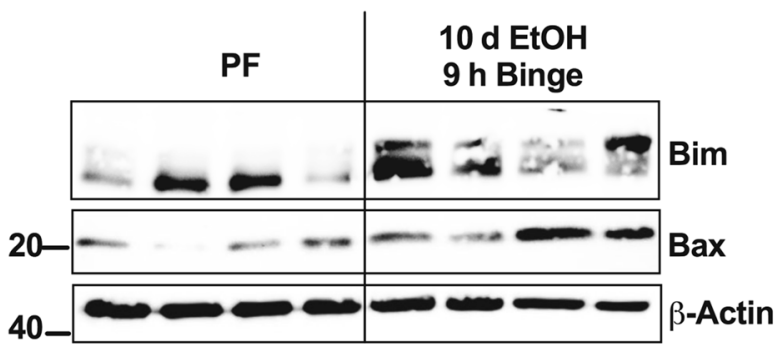

b CHOP in
epithelial
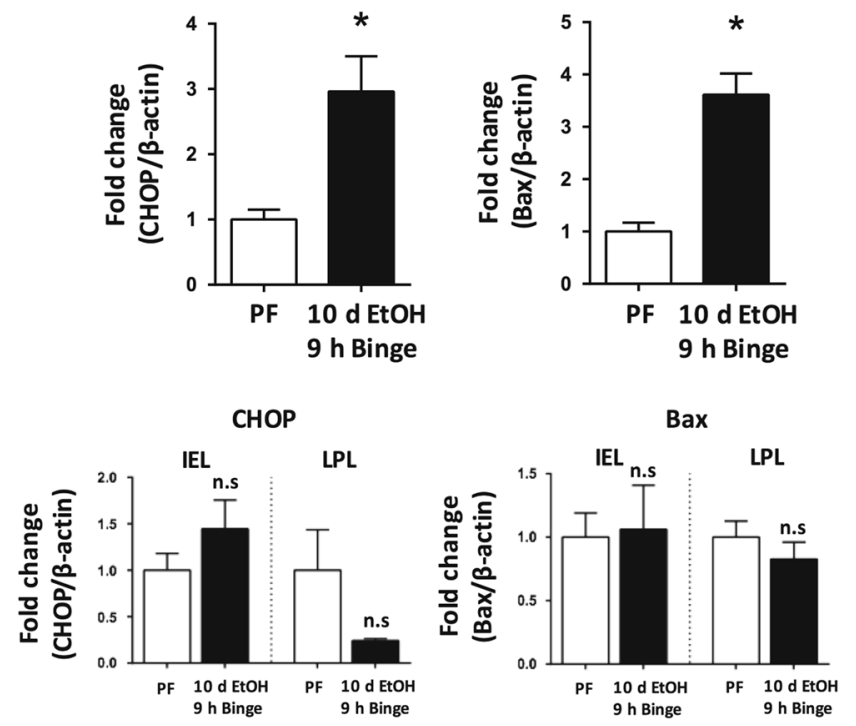

d
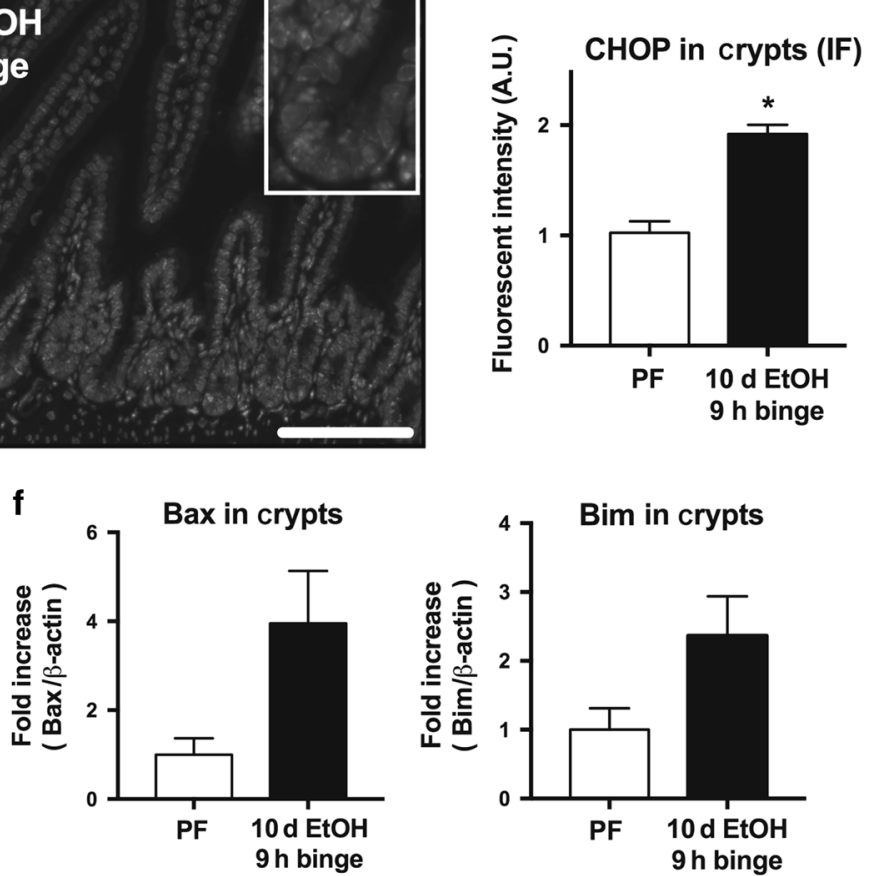

Bax in epithelial

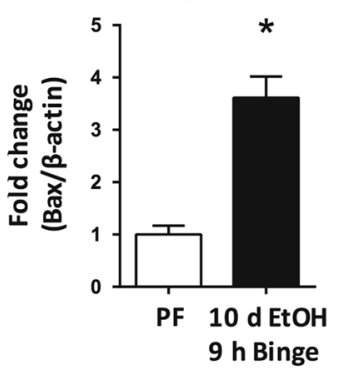

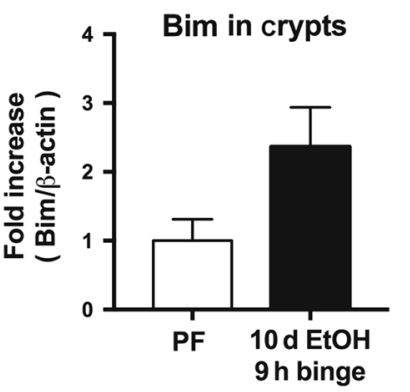

Fig. 6 Alcohol-induced CHOP expression localizes to Paneth cells. a PSI epithelial and immune cell fractions (intraepithelial [IEL] and lamina propria leukocytes [LPL]) were isolated from pair-fed (PF) and 10 day EtOH $9 \mathrm{~h}$ binge mice for Western blot analysis and quantified (b) CHOP and Bax proteins. c Representative CHOP immunofluorescent (IF) images of PSI samples of pair-fed (PF) and 10 day EtOH $9 \mathrm{~h}$ binge mice. d Insets show individual crypts and the more intense IF staining observed in the 10-day $+9 \mathrm{~h}$ binge alcohol samples. e Crypts were isolated from PF and 10 day EtOH $9 \mathrm{~h}$ binge mice and Bax and Bim proteins were measured by Western blot and quantified (f). $n=3-7$ mice/group; ${ }^{*} p<0.05$ vs. pair-fed. Scale bars $=50 \mu \mathrm{m}$

number of PCs increases from proximal to distal and expansion of the PC population was found in infection. ${ }^{27,28}$ Here, we discovered that alcohol selectively increased the abundance of PCs in the PSI, but not the DSI, in mice.

We also describe increased expression of genes involved in intestinal cell differentiation, such as Sox9, and its downstream target, Igfbp4. ${ }^{18}$ Spdef and Math1 are important for maintenance and differentiation of the PC lineage, ${ }^{19}$ while Lgr5 is a marker for intestinal stem cells. ${ }^{29}$ The expression of all of these factors was increased in alcohol-fed animals suggesting expansion of PCs. A recent study from Schmitt et al. demonstrated that intestinal inflammation, similar to that induced by alcohol, led to an increase in Paneth cell stemness and $P^{30}$ which may explain in part the paradoxical findings presented here that alcohol induces both cell death in the crypts of the PSI while also increasing Paneth cell numbers and expression of stem-related genes. Interestingly, 
a

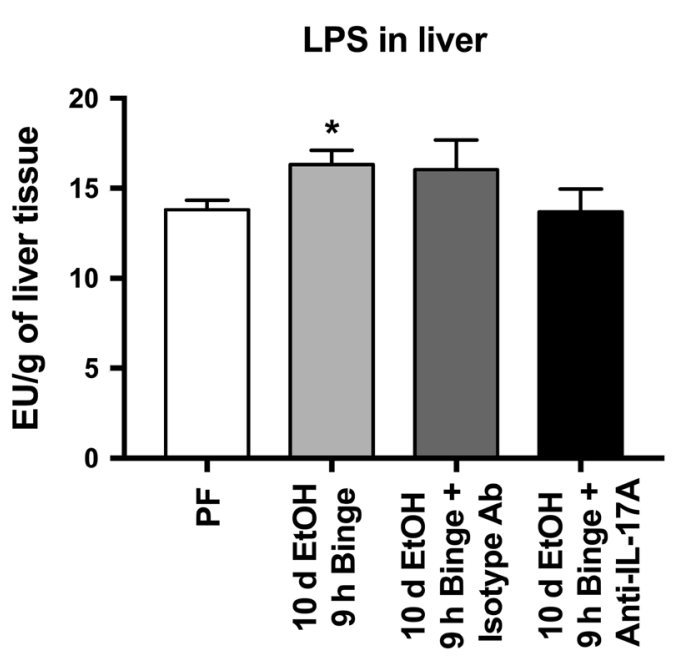

b
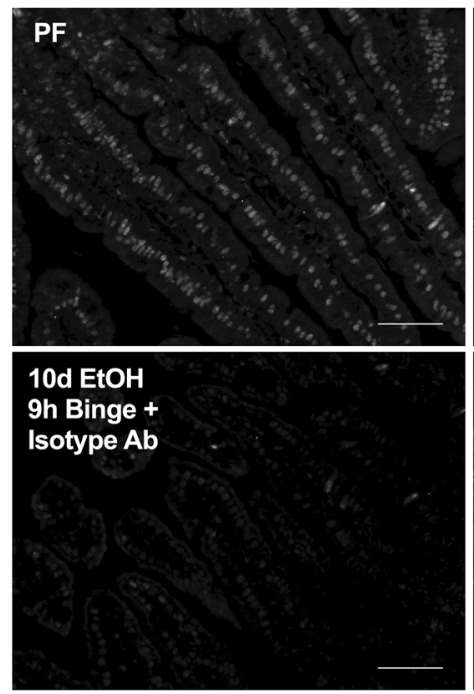
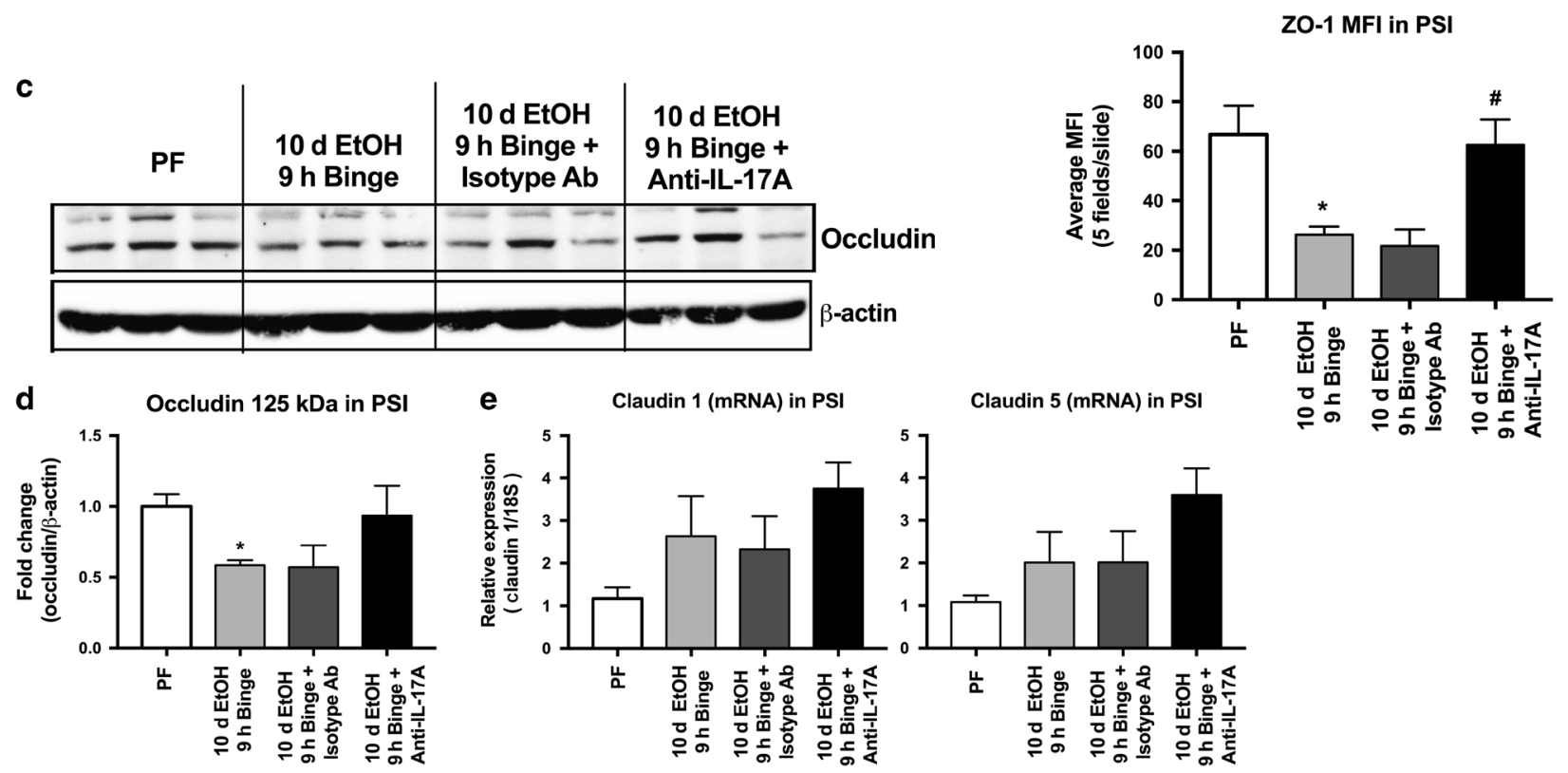

Claudin 15 (mRNA) in PSI

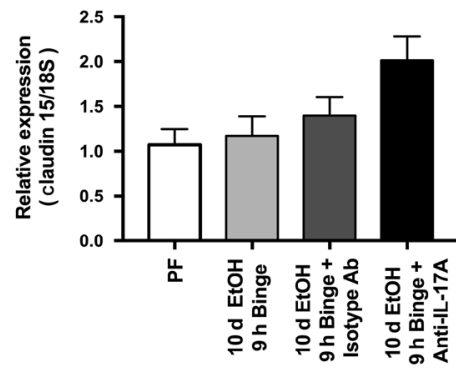

Occludin (mRNA) in PSI
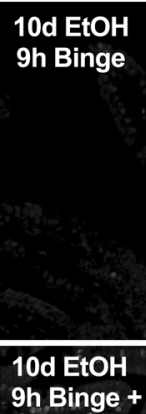

Anti-IL-17A

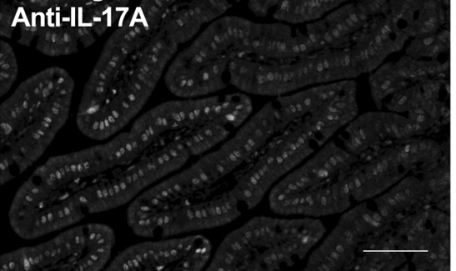

ZO-1 MFI in PSI
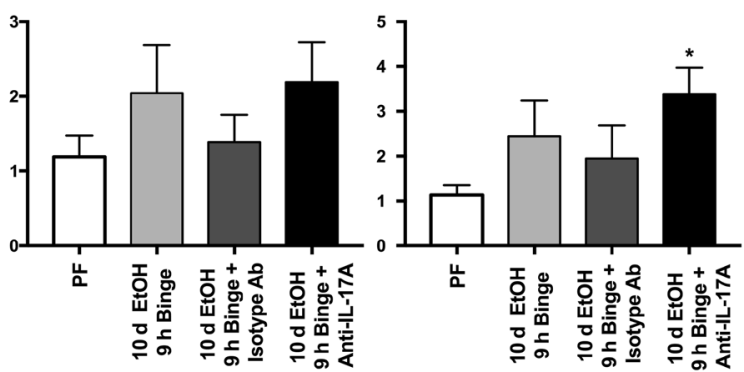

Fig. 7 Tight-junction levels in the proximal small intestine are reduced by alcohol and restored by in vivo IL-17 blockade. a The bacterial cell wall component LPS was measured in the livers of pair-fed (PF) and 10 day EtOH $9 \mathrm{~h}$ binge mice, as well as EtOH mice treated with anti-IL-17 antibody or an isotype control. b ZO-1 immunofluorescent (IF) staining was completed on the PSI of these same mice and quantified. c Occludin was measured from the PSI by Western blot and quantified (d). mRNA expression of multiple tight junction proteins including Claudin-1, Claudin-5, and Claudin-15, Occludin and ZO-1 were measured by qPCR from PSI. For a, $n=5-8$ mice/group. For B, $n=6$ mice/group and 5 images/mouse were quantified. For c-d, $n=6$ mice/group. For e, $n=7-10$ mice/group. ${ }^{*} p<0.05$ vs. pair-fed (PF) 


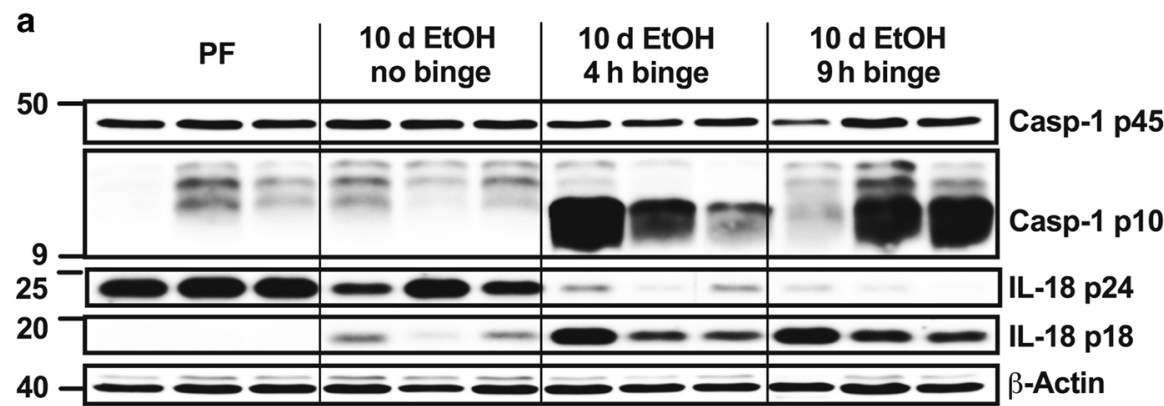

b

Caspase-1 p10/p45
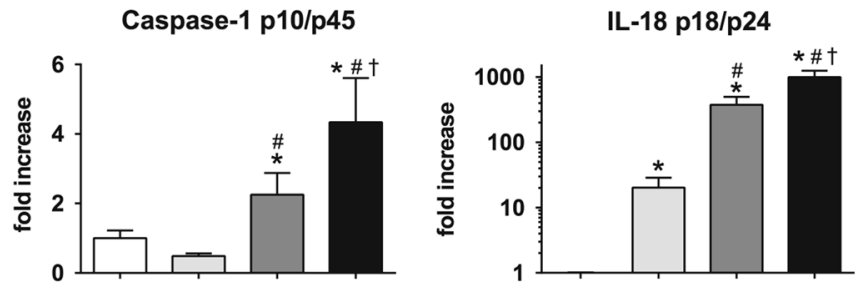

$\square \mathrm{PF}$

$10 \mathrm{~d}$ EtOH no binge

$10 \mathrm{~d}$ EtOH $4 \mathrm{~h}$ binge

$10 \mathrm{~d} \mathrm{EtOH} 9 \mathrm{~h}$ binge

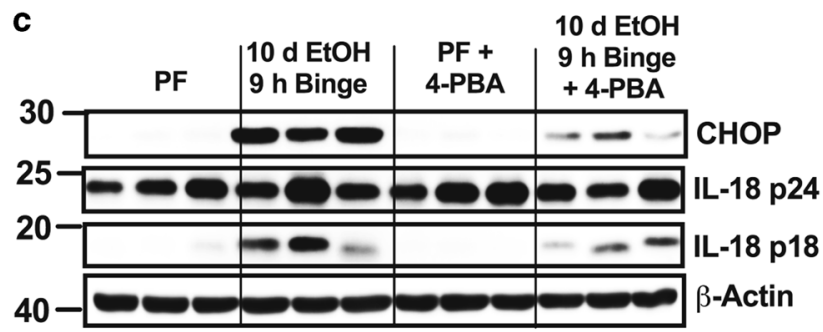

d
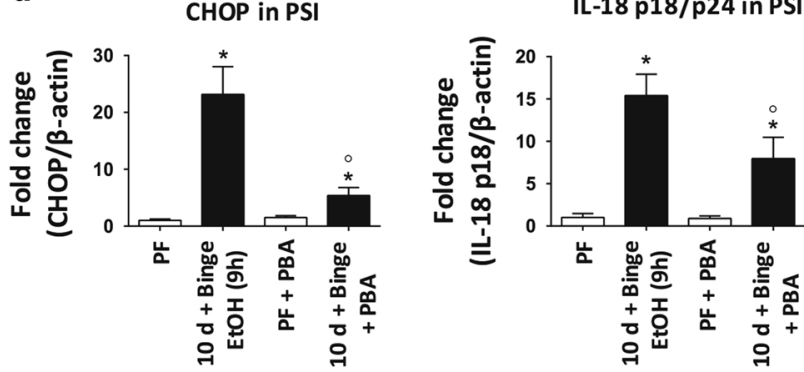

e

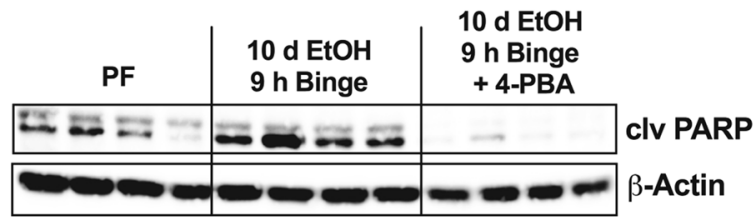

f

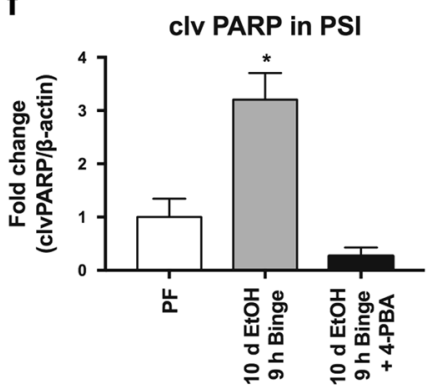

h

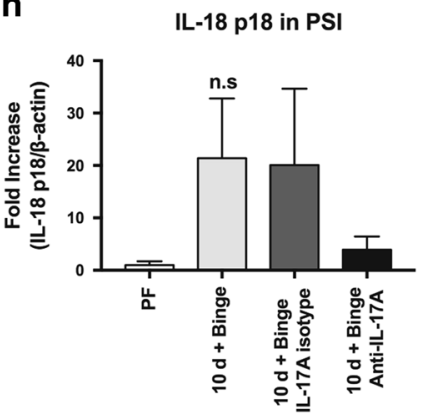

Fig. 8 Inflammasome activation by acute alcohol binge in the small intestine is attenuated by in vivo inhibition of ER stress. a Activation of inflammasome components caspase- 1 and IL-18, including the active cleaved forms Casp- 1 p10 and IL-18 p18, were assessed by Western blot and quantified (b) from the PSI of pair-fed (PF), chronic alcohol without binge, 10 day EtOH $4 \mathrm{~h}$ binge and 10 day EtOH $9 \mathrm{~h}$ binge mice. c ER stress inhibition through in vivo 4-phenylbutiric acid (4-PBA) administration was used to reduce CHOP expression and inflammasome target IL-18 activation was measured by Western blot and quantified (d) from PF and 10 day EtOH $9 \mathrm{~h}$ binge mice. e Western blot analysis and quantification (f) of cleaved (clv) PARP from PF and alcohol-fed mice with and without 4-PBA treatment. $\mathbf{g}$ Activation of IL-18 was measured by Western blot and quantified (h) in the PSI of PF and 10 day EtOH $9 \mathrm{~h}$ binge mice, as well as EtOH mice treated with anti-IL-17 antibody or an isotype control. $n=5-7$ mice/group; $p<0.05 *$ vs. pair-fed, \# vs. $10 \mathrm{~d}$ EtOH No binge, $\dagger$ vs. 10 day EtOH $4 \mathrm{~h}$ binge, ${ }^{\circ}$ vs. 10 day EtOH $9 \mathrm{~h}$ binge 
a

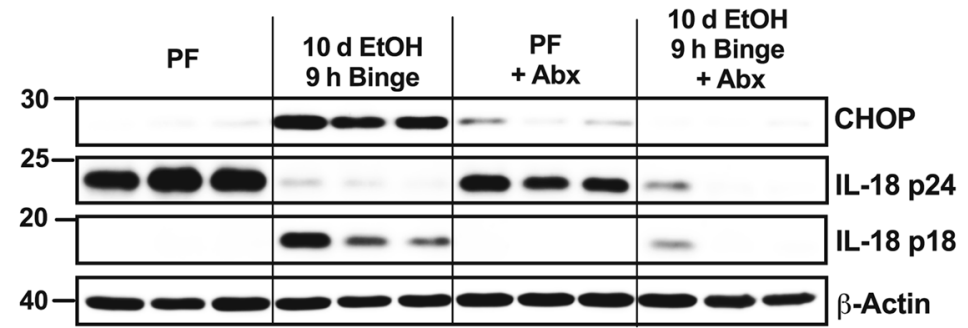

b

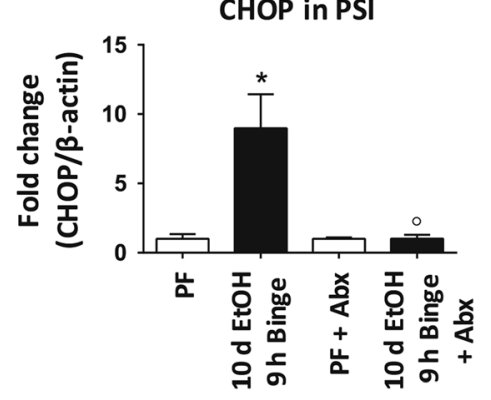

IL-18 p18/p24 in PSI

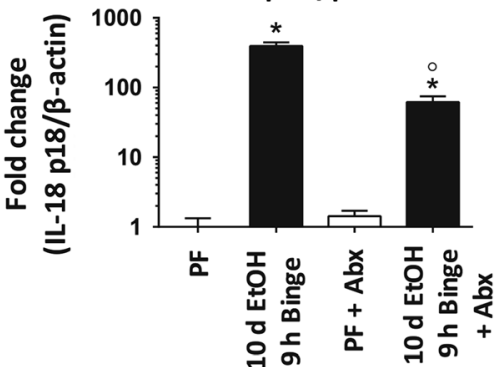

C

Lysozyme in PSI (IHC)
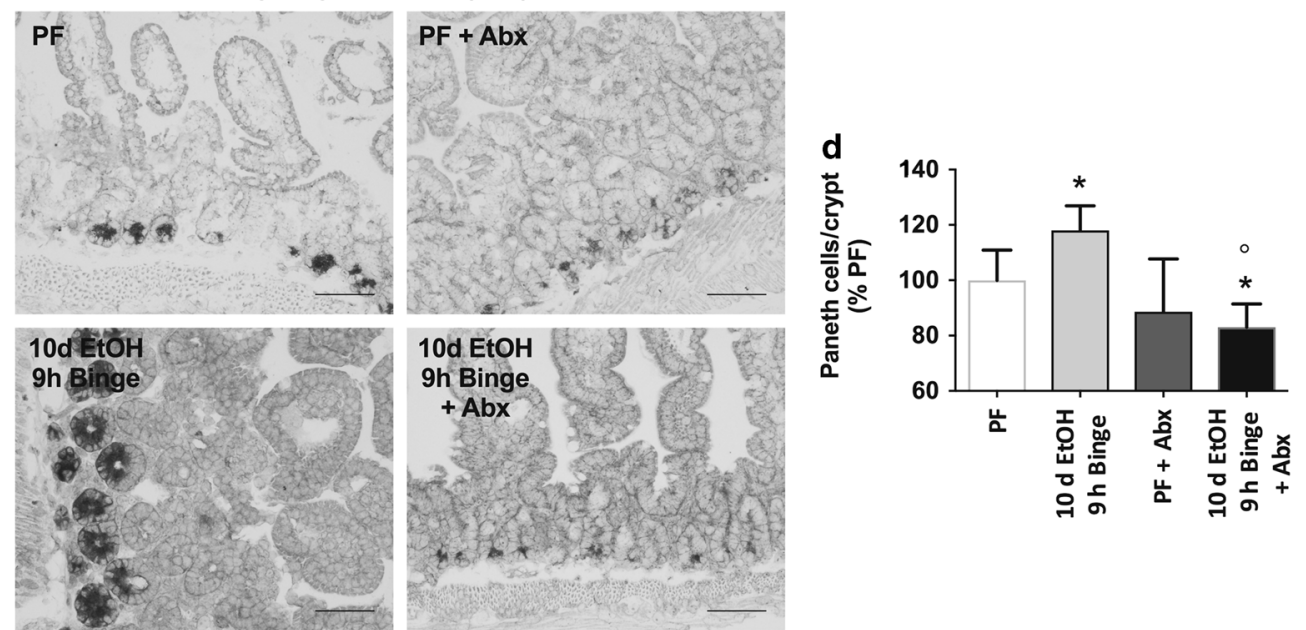

Fig. 9 Alcohol-induced CHOP induction, IL-18 activation and PC increases are mediated by the gut microbiota load. a Gut decontamination using broad-spectrum antibiotics administration (Abx; ampicillin, neomycin, vancomycin and metronidazole) significantly reduced the gut microbiota load. CHOP and IL-18 activation were measured from PSI of PF and 10 day EtOH $9 \mathrm{~h}$ binge mice by Western blot and quantified (b). c Representative images and (d) quantification of lysozyme immunohistochemistry (IHC) from PF and 10 day EtOH $9 \mathrm{~h}$ binge alcohol-fed mice. $n=6-10$ mice/group; $p<0.05 *{ }^{*}$ vs. pair-fed, ${ }^{\circ}$ vs. 10 day EtOH $9 \mathrm{~h}$ binge

PERK-elF2 $a$ dependent signaling plays a major role in intestinal stem cell differentiation ${ }^{31}$ and elF2a phosphorylation is required for normal PC function and mucosal homeostasis. ${ }^{32}$ Therefore, it is tempting to speculate that alcohol-induced elF2a phosphorylation contributes to increased PC numbers by inducing their differentiation. Other mechanisms, such as microbiota-mediated PC expansion, are also a possibility.

Strikingly, we found that ethanol stimulated IL-17A release in a dose-dependent manner in isolated crypts, mimicking our observations of increased IL-17 in vivo expression in alcohol-fed mice. IL-17A is a homeostatic cytokine in the gut that is constitutively present in PC granules ${ }^{33}$ and regulates tight junction barrier integrity. Consistent with this, we found that anti-IL-17 blocking in vivo prevented the alcohol-induced increase in LPS in the liver and restored the decreased expression of the tight junction proteins, ZO-1 and occludin, in the PSI in alcohol-fed mice indicating that mechanistically, IL-17 plays a role in increased gut permeability in alcoholic liver disease. This was also supported by our observation of upregulated pro-apoptotic molecules mediated by alcohol and IL-17 and the absence thereof in IL-17 antibody-treated mice.

PC-derived IL-17A has been implicated in distant organ injury, including hepatic ischemia/reperfusion ${ }^{8}$ and shock. ${ }^{33}$ In our experiments, the rapid release of IL-17A from ethanol-treated crypts and the low abundance of $\mathrm{IL}-17^{+}$immune cells suggest PCs, rather than immune cells, as the primary source of secreted IL-17A in vitro and in vivo. While our in vitro paradigm uses high concentrations of alcohol (4-100 mM) for multiple hour durations, conditions that may be supraphysiologic and difficult to reproduce in an in vivo model, our findings do emphasize the results from 10-day acute-on-chronic mouse model. Different factors including Toll-like receptor ligands, bacteria, TNFa, and immune cell-derived interferon- $\gamma$ have been shown to induce PC degranulation. ${ }^{34}$ Here, we show that IL-17A secretion was 
No alcohol

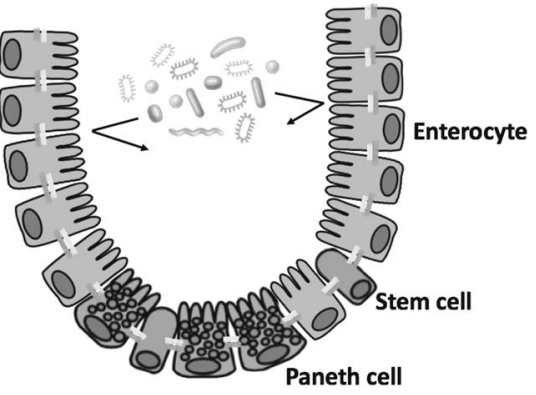

\section{$10 \mathrm{~d}$ EtOH binge}

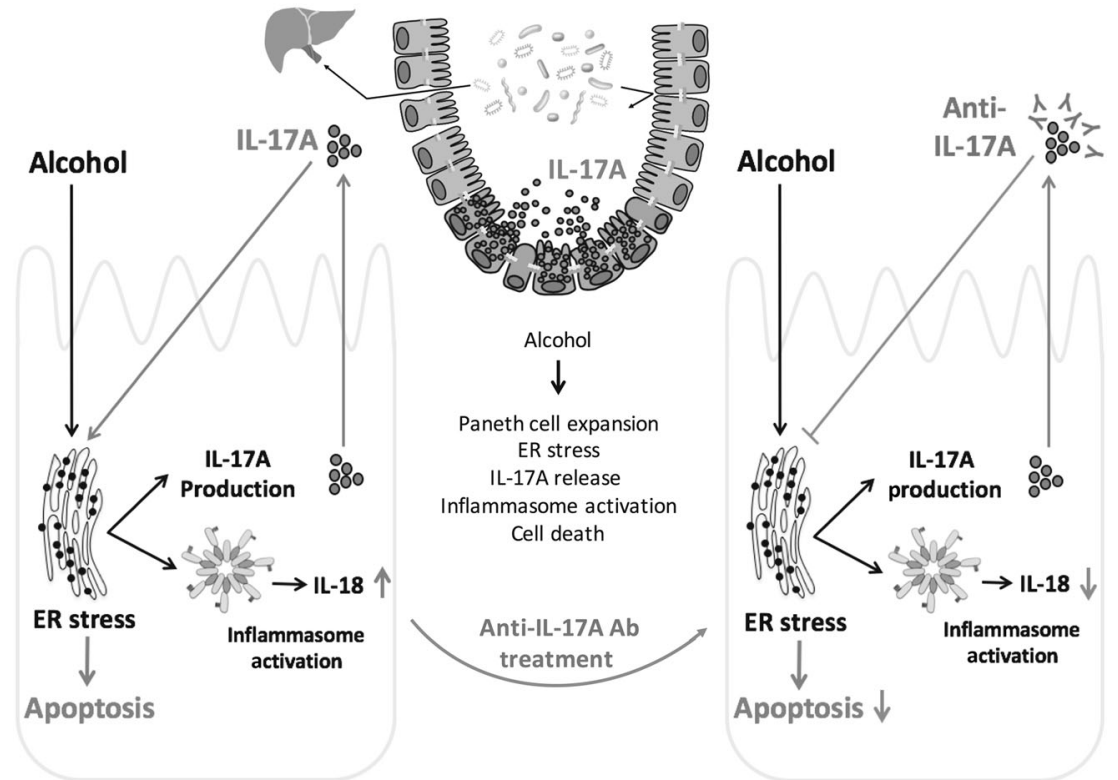

Fig. 10 Increased IL-17 production, Paneth cell activation, inflammasome and ER stress in the proximal small intestine after acute-on-chronic alcohol in mice. Working model based on our data indicates that acute binge on chronic alcohol exposure results in increased abundance of Paneth cells in the PSI, leading to ER stress induction, IL-17A release, apoptosis and inflammasome activation in crypts in the PSI. The mechanistic role of alcohol-induced IL-17A is indicated by experiments where IL-17 blocking antibody prevented alcohol-induced ER stress, apoptosis, inflammasome activation and IL-18 production in isolated crypts in vitro and in vivo in the PSI

mimicked by the ER stress inducer thapsigargin and alcoholinduced IL-17A was inhibited by the ER stress inhibitor 4-PBA, implicating alcohol-induced ER stress as a promoter of PC degranulation. In human alcoholic hepatitis, increased circulating IL-17 levels were found to be associated with an increase in Th17 cells in the livers of patients with alcoholic hepatitis. ${ }^{35}$ Here, we found no significant increase in liver IL-17A levels or in Th17 cells in the acute-on-chronic alcoholic liver disease model (data not shown). We speculate that the increase in IL-17A in PCs may be local mechanism in the PSI rather than a systemic effect promoting liver inflammation. ${ }^{15}$ ER stress is a wellcharacterized pathophysiological factor in intestinal inflammatory diseases ${ }^{24,36,37}$ and alcohol is a potent ER stressor. ${ }^{10}$ Although the mechanisms of alcohol-induced ER stress remain to be elucidated, we found that alcohol significantly increased elF2a phosphorylation and CHOP levels in the PSI. This suggests that acute-on-chronic alcohol may activate CHOP-mediated UPR signaling. ${ }^{38}$

Here, we show that upregulation of CHOP expression in the proximal (but not in the distal) SI of alcohol-fed mice overlapped with the cellular distribution of PCs. Previous studies identified PCs as a main target of ER stress signaling and described PCs as one of the most susceptible intestinal epithelial cell types for perturbations of ER homeostasis. ${ }^{39}$ PERK and downstream phosphorylation of eukaryotic translation initiation factor $2 a$ (elF2a) play a physiological role in intestinal stem cell differentiation. ${ }^{31}$ Moreover, the p-elF2a arm of the UPR is required for the normal function of PCs and mucosal homeostasis. ${ }^{32}$ Interestingly, bacteria-derived factors have been shown to induce UPR signaling and subsequent inflammation, ${ }^{40}$ while administration of the ER stress inhibitor, 4-phenylbutyric acid (4-PBA), ameliorated dextran sulfate sodium (DSS)-induced colitis $^{22}$ similar to the effect we observed with alcohol feeding.

We found that reducing gut bacterial load through antibiotic administration prevented CHOP induction in vivo. Chronic alcohol ingestion is associated with both quantitative (small intestinal bacterial overgrowth) and qualitative (dysbiosis) microbiome changes in the SI and PC antimicrobial peptide production is highly inducible by the microbiome. ${ }^{41}$ Thus, changes in the PSI microbiome might be an indirect mechanism by which alcohol affects PCs. Importantly, alcohol induces changes in the microbiome throughout the intestinal tract while our present study focuses only on SI. Interestingly, PCs express ethanol-metabolizing enzymes and are prone to acetaldehyde accumulation due to lowered acetaldehyde oxidizing capacity. ${ }^{42}$ Acetaldehyde is a potent ER stressor through adduct formation and by impairing 
protein glycosylation. Thus, ethanol metabolites in PCs could directly cause ER stress.

Prolonged and/or unresolved ER stress leads to apoptosis. While the chronic alcohol feeding alone did not induce cell death signaling, the addition of an acute binge increased pro-apoptotic signaling with phosphorylation of elF2a and induction of CHOPrelated downstream targets, GADD34, Bax, and Bim. Phosphorylation of elF2a decreases protein load in the cell by attenuating translation, while allowing expression of certain ER stress-related proteins. $^{12}$ Removing p-elF2a-mediated translational block by GADD34, a downstream target of CHOP, allows for further synthesis of pro-apoptotic molecules. ${ }^{12}$ Indeed, we found increased expression of the small mitochondrial pore-forming proteins, Bim and Bax, in the PSI of alcohol-fed animals. Overexpression of CHOP induces apoptosis by enhancing activation and mitochondrial translocation of $\mathrm{Bax}^{43}$ and induces Bim expression, ${ }^{44}$ eventually leading to mitochondrial membrane destabilization and downstream caspase activation. We show increased cleavage of the executioner caspase, caspase- 3 , and its downstream target PARP, both well-known markers of ongoing apoptotic signaling. Thus, we conclude that acute-on-chronic alcohol activates the CHOP-mediated mitochondrial apoptosis pathway leading to downstream caspase activation.

PCs are a site of origin for intestinal inflammation, ${ }^{24}$ therefore we sought to identify possible pro-inflammatory pathways. In line with our previous data in the liver ${ }^{45,46}$ and intestine, ${ }^{2}$ we found that alcohol induces increased expression of the pro-inflammatory cytokines including Tnfa, II-6, II1 $\beta$, Mcp1, II23, and II17 in the PSI. Now we report increased activation of the inflammasome upon acute-on-chronic alcohol ingestion indicated by activation of the caspase-1 inflammasome and cleavage of its target, IL-18. DupaulChicoine et al. recently showed that caspase- 1 knockout mice were more susceptible to acute DSS-induced colitis and exogenous IL-18 administration resulted in protection in this model. ${ }^{47}$ Zaki et al. found increased IL-18 production in DSS-induced colitis and suggested that epithelial inflammasome activation was followed by a regenerative proliferative response. ${ }^{48}$ Further, these studies identified epithelial cells as the main source of IL-18 production.

Recently, caspase-2-mediated activation of the pro-apoptotic molecule, Bid, was shown to link ER stress to inflammasome activation. ER stress induced truncation of Bid through caspase-2 activation, which in turn translocated to the mitochondrial membrane leading to the release of mitochondrial content and inflammasome activation. ${ }^{13}$ Further, Upton et al. showed that ER stress increased caspase-2 levels by downregulating inhibitory microRNA expression leading to apoptosis. ${ }^{49}$ In our study, we found significant upregulation of both caspase-2 and Bid protein expression along with increased cleaved form of Bid, tBid. In addition, caspase-8, the downstream effector of the extrinsic apoptotic pathway that can cleave Bid, ${ }^{50}$ was induced by acute-on-chronic alcohol in the PSI. This suggests that alcohol might independently induce both extrinsic and intrinsic apoptotic pathways, converging on mitochondrial membrane destabilization.

Importantly, our study focused on the PSI where we noted a significant effect of alcohol on PCs, inflammatory cytokines, ER stress and tight junction protein. However, we did not investigate each of these factors throughout the entire gastrointestinal system, including the DSI (for some markers) and colon. Future investigation of these segments, and the regional effect of alcohol on gut barrier function, immune signaling and cellular response may provide additional information on the role of PC in alcoholinduced gut permeability and ALD.

In conclusion, we describe a novel, alcohol-induced proapoptotic signaling and inflammasome activation in the small intestine. We show that alcohol upregulates PC numbers in the PSI and that alcohol primarily affects PCs, which respond with IL-17A release in vitro. Our findings underscore the importance of examining the PSI not only as a microbiota-reservoir and tight junction barrier, but also as a complex organ with multiple response mechanisms to various impacts, specifically alcoholmediated injuries.

\section{MATERIALS AND METHODS}

Animal experiments

This study was conducted according to the regulations of the University of Massachusetts Medical School IACUC (Worcester, MA). Eight-week-old to ten-week-old, female, wild-type C57BL/6 mice (Jackson Laboratory, Bar Harbor, ME) were fed a 5\% (vol/vol) ethanol containing Lieber-DeCarli liquid diet or calorie-matched diet for 10 days with or without an oral gavage of $5 \mathrm{~g} / \mathrm{kg}$ ethanol or maltose dextrin solution, respectively (the NIAAA mode ${ }^{15}$ ). Mice were sacrificed 4 or $9 \mathrm{~h}$ after the gavage. For depletion of intestinal microbiota, ampicillin $(1 \mathrm{mg} / \mathrm{ml})$, neomycin $(10 \mathrm{mg} / \mathrm{ml})$, vancomycin $(5 \mathrm{mg} / \mathrm{ml})$ and metronidazole $(10 \mathrm{mg} / \mathrm{ml})$ were administered by gavage twice daily 5 days prior to and throughout the experiment. To verify successful decontamination of the gut, feces was cultured under aerobic and anaerobic conditions. 4-PBA (Calbiochem; $1 \mathrm{~g} / \mathrm{kg}$ mouse/day) was dissolved in water and mixed with the liquid diet. Its concentration in the diet was calculated based on daily monitoring of diet consumption (average $15 \mathrm{ml} /$ day). IL-17A blocking antibody (R\&D) was injected three times before binge alcohol gavage; on 1st and 6th day of $5 \%$ alcohol diet, 50ug of IL-17A blocking or isotype antibody was injected per mouse through i.p. injection, and 100ug of each antibody was injected on the 10th day of $5 \%$ alcohol diet for the last treatment $3 \mathrm{~h}$ before binge alcohol gavage.

Sample collection, ELISA, Immunoblotting, GRT-PCR reaction and histology are detailed in the Supplementary Materials and Methods.

\section{Statistical analysis}

Data are shown as mean \pm standard error of the mean. Statistical tests included Mann-Whitney or unpaired student $t$-test and $p<0.05$ was considered statistically significant. Graphpad prism software was used to perform Grubbs test to remove outlier. $\left(^{*}=0.05\right)$.

\section{ACKNOWLEDGEMENTS}

The authors thank Karen Kodys and Donna Catalano for their technical assistance. The authors also thank Frances S. Brown, Dr. Arlin B. Rogers, and the Cummings School of Veterinary Medicine at Tufts University for their excellent technical assistance and expert analysis of histology samples. We are especially thankful for assistance of Candice Dufour and Melanie Trombly for their assistance with proofreading, preparation, and uploading of the manuscript. This work was supported by an NIH grant from the NIAAA; R01 AA017729 (to G.S.), F30 AA024680 (to P.L.), F31 AA025545 (to A.I.V.) and F30 AA022283 (to A.S.).

\section{AUTHOR CONTRIBUTIONS}

B.G., Y.C. and G.S. conceived and designed the experiments; B.G., P.L., Y.C., C.D.C. performed the experiments with assistance from AIV, AS and A.A.; B.G., Y.C., P.L., C.D. C., A.I.V. and G.S. analyzed the data; B.G., P.L. and G.S. wrote the paper; P.L., A.I.V., A.S. and G.S. obtained funding and G.S. supervised the project.

\section{ADDITIONAL INFORMATION}

The online version of this article (https://doi.org/10.1038/s41385-019-0170-4) contains supplementary material, which is available to authorized users.

Competing interests: G.S. has received funding from NIH-NIAAA, Gilead, Genfit, University of Florida, Intercept, Allergan, Novartis, SignaBlok and Shire. The rest of the authors declare no competing interests.

Publisher's note: Springer Nature remains neutral with regard to jurisdictional claims in published maps and institutional affiliations. 


\section{REFERENCES}

1. Hartmann, P., Seebauer, C. T. \& Schnabl, B. Alcoholic liver disease: the gut microbiome and liver cross talk. Alcohol. Clin. Exp. Res. 39, 763-775 (2015).

2. Lippai, D., Bala, S., Catalano, D., Kodys, K. \& Szabo, G. Micro-RNA-155 deficiency prevents alcohol-induced serum endotoxin increase and small bowel inflammation in mice. Alcohol. Clin. Exp. Res. 38, 2217-2224 (2014).

3. Lowe, P. P. et al. Alcohol-related changes in the intestinal microbiome influence neutrophil infiltration, inflammation and steatosis in early alcoholic hepatitis in mice. PLOS ONE 12, e0174544 (2017).

4. Szabo, G. \& Lippai, D. Converging actions of alcohol on liver and brain immune signaling. Int. Rev. Neurobiol. 118, 359-380 (2014).

5. Bevins, C. L. \& Salzman, N. H. Paneth cells, antimicrobial peptides and maintenance of intestinal homeostasis. Nat. Rev. Microbiol. 9, 356-368 (2011).

6. Clevers, H. C. \& Bevins, C. L. Paneth cells: maestros of the small intestinal crypts. Annu. Rev. Physiol. 75, 289-311 (2013).

7. Lee, J. S. et al. Interleukin-23-independent IL-17 production regulates intestinal epithelial permeability. Immunity 43, 727-738 (2015).

8. Park, S. W., Kim, M., Brown, K. M., D'Agati, V. D. \& Lee, H. T. Paneth cell-derived interleukin-17A causes multiorgan dysfunction after hepatic ischemia and reperfusion injury. Hepatology 53, 1662-1675 (2011).

9. Hosomi, S., Kaser, A. \& Blumberg, R. S. Role of endoplasmic reticulum stress and autophagy as interlinking pathways in the pathogenesis of inflammatory bowel disease. Curr. Opin. Gastroenterol. 31, 81-88 (2015).

10. Ji, C. Mechanisms of alcohol-induced endoplasmic reticulum stress and organ injuries. Biochem. Res. Int. 2012, 216450 (2012).

11. Bettigole, S. E. \& Glimcher, L. H. Endoplasmic reticulum stress in immunity. Annu. Rev. Immunol. 33, 107-138 (2015).

12. Szegezdi, E., Logue, S. E., Gorman, A. M. \& Samali, A. Mediators of endoplasmic reticulum stress-induced apoptosis. EMBO Rep. 7, 880-885 (2006).

13. Bronner, D. N. et al. Endoplasmic reticulum stress activates the inflammasome via NLRP3- and Caspase-2-driven mitochondrial damage. Immunity 43, 451-462 (2015).

14. Szabo, G. \& Petrasek, J. Inflammasome activation and function in liver disease. Nat. Rev. Gastroenterol. Hepatol. 12, 387-400 (2015).

15. Bertola, A., Mathews, S., Ki, S. H., Wang, H. \& Gao, B. Mouse model of chronic and binge ethanol feeding (the NIAAA model). Nat. Protoc. 8, 627-637 (2013).

16. Van Dussen, K. L., Dempsey, P. J., Samuelson, L. C. Chapter 11-Notch Pathway Regulation of Intestinal Cell Fate A2-Johnson, Leonard R. In Physiology of Gastrointestinal Tract 5th edn. (eds. Ghishan F. K., Kaunitz J. D., Merchant J. L., Said H. M., Wood J. D.) 329-357 (Academic Press, Boston, 2012).

17. Gassler, N. Paneth cells in intestinal physiology and pathophysiology. World J. Gastrointest. Pathophysiol. 8, 150-160 (2017).

18. Bastide, P. et al. Sox 9 regulates cell proliferation and is required for Paneth cell differentiation in the intestinal epithelium. J. Cell Biol. 178, 635-648 (2007).

19. Gregorieff, A. et al. The ets-domain transcription factor Spdef promotes maturation of goblet and Paneth cells in the intestinal epithelium. Gastroenterology 137, 1333-1345 (2009). e1331-1333.

20. $\mathrm{Yu}, \mathrm{T}$. et al. Kruppel-like factor 4 regulates intestinal epithelial cell morphology and polarity. PloS ONE 7, e32492 (2012).

21. Maloy, K. J. \& Kullberg, M. C. IL-23 and Th17 cytokines in intestinal homeostasis. Mucosal Immunol. 1, 339-349 (2008).

22. Cao, S. S. et al. The unfolded protein response and chemical chaperones reduce protein misfolding and colitis in mice. Gastroenterology 1s44, 989-1000 e1006 (2013).

23. Hetz, C. The unfolded protein response: controlling cell fate decisions under ER stress and beyond. Nat. Rev. Mol. Cell Biol. 13, 89-102 (2012).

24. Adolph, T. E. et al. Paneth cells as a site of origin for intestinal inflammation. Nature 503, 272-276 (2013).

25. Lowe, P. P. et al. Reduced gut microbiome protects from alcohol-induced neuroinflammation and alters intestinal and brain inflammasome expression. J. Neuroinflamm. 15, 298 (2018).
26. Purohit, V. et al. Alcohol, intestinal bacterial growth, intestinal permeability to endotoxin, and medical consequences: summary of a symposium. Alcohol $\mathbf{4 2 ,}$ 349-361 (2008).

27. Martinez Rodriguez, N. R. et al. Expansion of Paneth cell population in response to enteric Salmonella enterica serovar Typhimurium infection. Infect. Immun. 80, 266-275 (2012)

28. Yan, A. W. et al. Enteric dysbiosis associated with a mouse model of alcoholic liver disease. Hepatology 53, 96-105 (2011).

29. Sato, T. et al. Paneth cells constitute the niche for Lgr5 stem cells in intestinal crypts. Nature 469, 415-418 (2011).

30. Schmitt, M. et al. Paneth cells respond to inflammation and contribute to tissue egeneration by acquiring stem-like features through SCF/c-Kit signaling. Cell Rep. 24, 2312-2328.e2317 (2018)

31. Heijmans, J. et al. ER stress causes rapid loss of intestinal epithelial stemness through activation of the unfolded protein response. Cell Rep. 3, 1128-1139 (2013).

32. Cao, S. S. et al. Phosphorylation of elF2alpha is dispensable for differentiation but required at a posttranscriptional level for paneth cell function and intestinal homeostasis in mice. Inflamm. Bowel Dis. 20, 712-722 (2014).

33. Takahashi, N. et al. IL-17 produced by Paneth cells drives TNF-induced shock. J. Exp. Med. 205, 1755-1761 (2008).

34. Farin, H. F. et al. Paneth cell extrusion and release of antimicrobial products is directly controlled by immune cell-derived IFN-gamma. J. Exp. Med. 211, 1393-1405 (2014).

35. Lemmers, A. et al. The interleukin-17 pathway is involved in human alcoholic liver disease. Hepatology 49, 646-657 (2009).

36. Grootjans, J. et al. Level of activation of the unfolded protein response correlates with Paneth cell apoptosis in human small intestine exposed to ischemia/ reperfusion. Gastroenterology 140, 529-539 e523 (2011).

37. Deuring, J. J. et al. Genomic ATG16L1 risk allele-restricted Paneth cell ER stress in quiescent Crohn's disease. Gut 63, 1081-1091 (2014).

38. DuRose, J. B., Tam, A. B. \& Niwa, M. Intrinsic capacities of molecular sensors of the unfolded protein response to sense alternate forms of endoplasmic reticulum stress. Mol. Biol. Cell 17, 3095-3107 (2006).

39. Kaser, A. et al. XBP1 links ER stress to intestinal inflammation and confers genetic risk for human inflammatory bowel disease. Cell 134, 743-756 (2008).

40. Pillich, H., Loose, M., Zimmer, K. P. \& Chakraborty, T. Diverse roles of endoplasmic reticulum stress sensors in bacterial infection. Mol. Cell. Pediatr. 3, 9 (2016).

41. Vaishnava, S., Behrendt, C. L., Ismail, A. S., Eckmann, L. \& Hooper, L. V. Paneth cells directly sense gut commensals and maintain homeostasis at the intestinal hostmicrobial interface. Proc. Natl Acad. Sci. USA 105, 20858-20863 (2008).

42. Chiang, C. P. et al. Expression pattern, ethanol-metabolizing activities, and cellular localization of alcohol and aldehyde dehydrogenases in human small intestine. Alcohol., Clin. Exp. Res. 36, 2047-2058 (2012).

43. Gotoh, T., Terada, K., Oyadomari, S. \& Mori, M. hsp70-DnaJ chaperone pair prevents nitric oxide- and CHOP-induced apoptosis by inhibiting translocation of Bax to mitochondria. Cell Death Differ. 11, 390-402 (2004).

44. Puthalakath, $\mathrm{H}$. et al. ER stress triggers apoptosis by activating $\mathrm{BH} 3$-only protein Bim. Cell 129, 1337-1349 (2007).

45. Ambade, A. et al. Pharmacological inhibition of CCR2/5 signaling prevents and reverses alcohol-induced liver damage, steatosis and inflammation in mice. Hepatology 69, 1105-1121 (2018).

46. Petrasek, J. et al. IL-1 receptor antagonist ameliorates inflammasome-dependent alcoholic steatohepatitis in mice. J. Clin. Investig. 122, 3476-3489 (2012).

47. Dupaul-Chicoine, J. et al. Control of intestinal homeostasis, colitis, and colitisassociated colorectal cancer by the inflammatory caspases. Immunity 32, 367-378 (2010).

48. Zaki, M. H. et al. The NLRP3 inflammasome protects against loss of epithelial integrity and mortality during experimental colitis. Immunity 32, 379-391 (2010).

49. Upton, J. P. et al. IRE1alpha cleaves select microRNAs during ER stress to derepress translation of proapoptotic Caspase-2. Science 338, 818-822 (2012).

50. Kantari, C. \& Walczak, H. Caspase- 8 and bid: caught in the act between death receptors and mitochondria. Biochim. et Biophys. Acta 1813, 558-563 (2011). 